1 Supplementary Information for

\title{
2 An N,S-anchored Single-atom Catalyst Derived 3 from Domestic Waste for Environmental 4 Remediation
}

5 Peixin Cui ${ }^{1}$, Qiang Yang ${ }^{1,4}$, Cun Liu ${ }^{l}, Y u$ Wang $^{3}$, Guodong Fang ${ }^{l}$, Dionysios D.

6 Dionysiou ${ }^{5}$, Tongliang $\mathrm{Wu}^{l}$, Yiyi Zhou ${ }^{1,2}$, Junxiang Ren ${ }^{1,2}$, Hongbo Hou ${ }^{4}$, Yujun Wang ${ }^{1,2 *}$

7 1. Key Laboratory of Soil Environment and Pollution Remediation, Institute of Soil

8 Science, Chinese Academy of Sciences, 210008 Nanjing, PR China

9 2. University of Chinese Academy of Science, 100049 Beijing, PR China

10 3. Shanghai Synchrotron Radiation Facility, Shanghai Advanced Research Institute,

11 Chinese Academy of Sciences, 201204 Shanghai, PR China

12 4. College of Environmental Science and Engineering, Central South University of

13 Forestry and Technology, 410004 Changsha, PR China

14 5. Environmental Engineering and Science Program, Department of Chemical and

15 Environmental Engineering (ChEE), University of Cincinnati, Cincinnati, 45221-0071

16 Ohio, USA

$17 *$ Corresponding author

18 E-mail address: yjwang@issas.ac.cn 
Figure S7. Shell-by-shell fitting of $\mathrm{k}^{3}$-weighted Fourier transformed Co K-edge EXAFS of Co

Figure S10. Effect of different catalyst dosages on PCB28 degradation .15

Figure S11. Effect of different PMS dosages on PCB28 degradation .16

Figure S13. Effect of different $\mathrm{Cl}^{-}$dosages on PCB28 degradation

Figure S14. Effect of different $\mathrm{HCO}_{3}{ }^{-}$dosages on $\mathrm{PCB} 28$ degradation

Figure S23. Shell-by-shell fitting of $\mathrm{k}^{3}$-weighted Fourier transformed Co K-edge EXAFS of Co-CGBC after activation reaction

Figure S24. The binding energies calculated by DFT with different coordination structures

52 Table S1. The basic properties of spent coffee ground and Co-CGBC .....................................30

53 Table S2. EXAFS fitting parameters at the Co K-edge for various samples.............................31

54 Table S3. The basic properties of the actual water from Xuanwu Lake........ . .32 
55 Table S4. Comparison of other Co-based catalysts for AOPs and pollutant degradation .33

56 Supplementary references .34

57

58 


\section{Text S1}

60 Chemicals. Potassium peroxymonosulfate $(99.5 \%), 2,4,,4^{\prime}$-trichlorobiphenyl (PCB28, $6199.5 \%$,), bisphenol A (BPA, >97\%) and diethyl phthalate (DEP, 99\%) were provided by 62 Alfa Aesar Co., Inc. (China). 5,5-Dimethyl-1-pyrroline N-oxide (DMPO), 4-hydroxy63 2,2,6,6-tetramethyl-piperidinol (TEMP) and other reagents of analytical grade, including 64 cobalt chloride hexahydrate $\left(\mathrm{CoCl}_{2} \cdot 6 \mathrm{H}_{2} \mathrm{O}\right)$, sodium hydroxide $(\mathrm{NaOH})$, ethanol, tertbutyl 65 alcohol (TBA), furfuryl alcohol (FFA), sodium dihydrogen phosphate $\left(\mathrm{NaH}_{2} \mathrm{PO}_{4}\right)$, sodium 66 hydrogen phosphate $\left(\mathrm{Na}_{2} \mathrm{HPO}_{4}\right)$, sodium carbonate $\left(\mathrm{Na}_{2} \mathrm{CO}_{3}\right)$, sodium iodide dihydrate $67\left(\mathrm{NaI} \cdot 2 \mathrm{H}_{2} \mathrm{O}\right)$, sodium chloride $(\mathrm{NaCl})$, and humic acid $(\mathrm{HA})$ were obtained from Sigma68 Aldrich. All chemicals were used without further purification. Ultra-pure water from Milli69 Q apparatus (Millipore, USA) was used in the experiments. 


\section{Text S2}

73 XANES calculation. The quantitative analysis of XANES spectra was performed using

74 the MXAN package, which is capable of quantitatively analyzing the XANES spectrum

75 from the absorption edge up to $\sim 200 \mathrm{eV}$ via comparison between experimental data and

76 theoretical calculations obtained by changing relevant geometrical parameters around the

77 photon absorber site. ${ }^{1-2}$ The X-ray absorption cross-sections were calculated using the full

78 multiple scattering approach in the framework of the muffin-tin (MT) approximation for

79 the shape of the potential. ${ }^{3-4}$ In this case, spectra typically converge when the cluster

80 includes 25 atoms within a radius of $5.0 \AA$.

81 


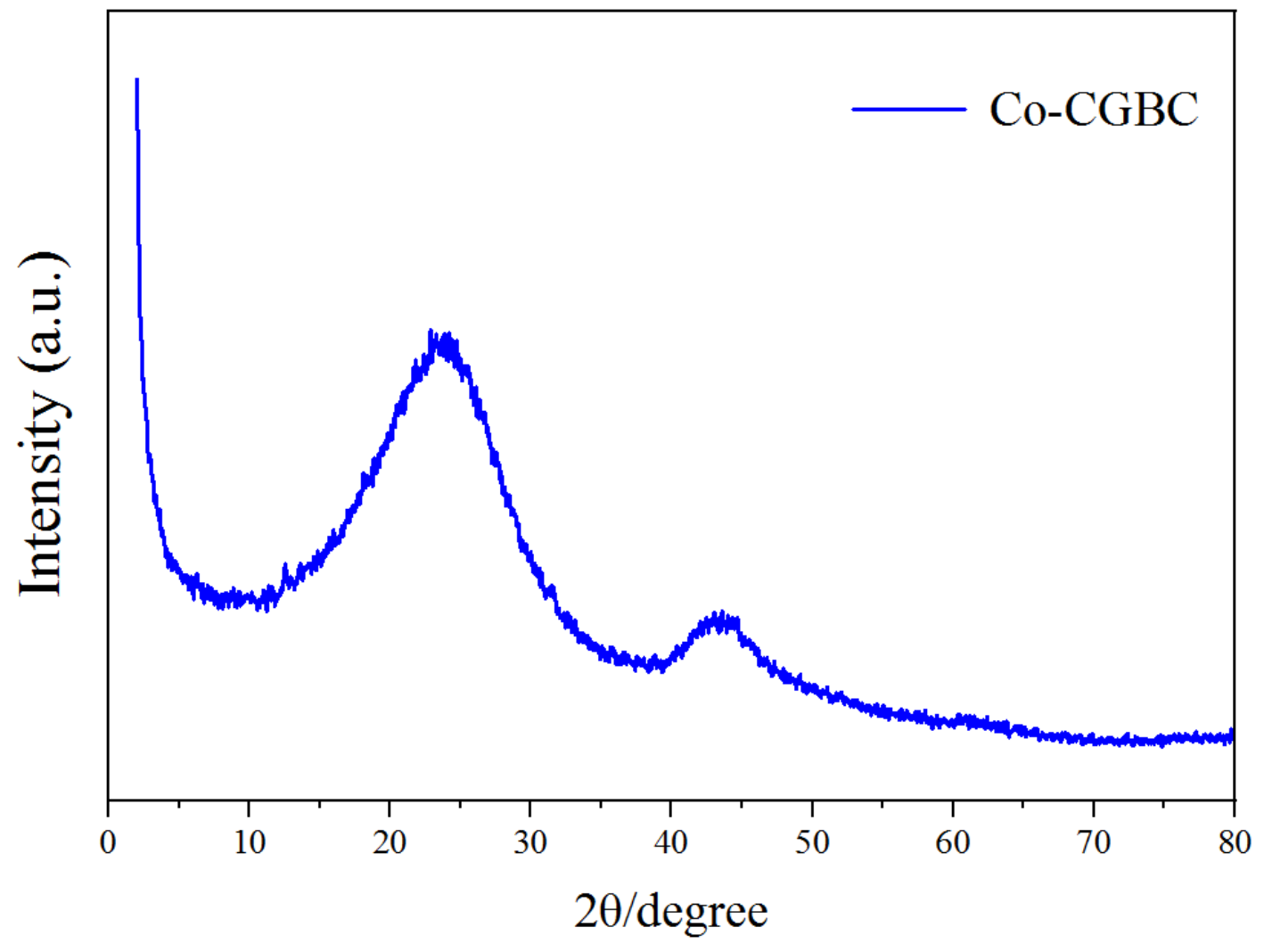




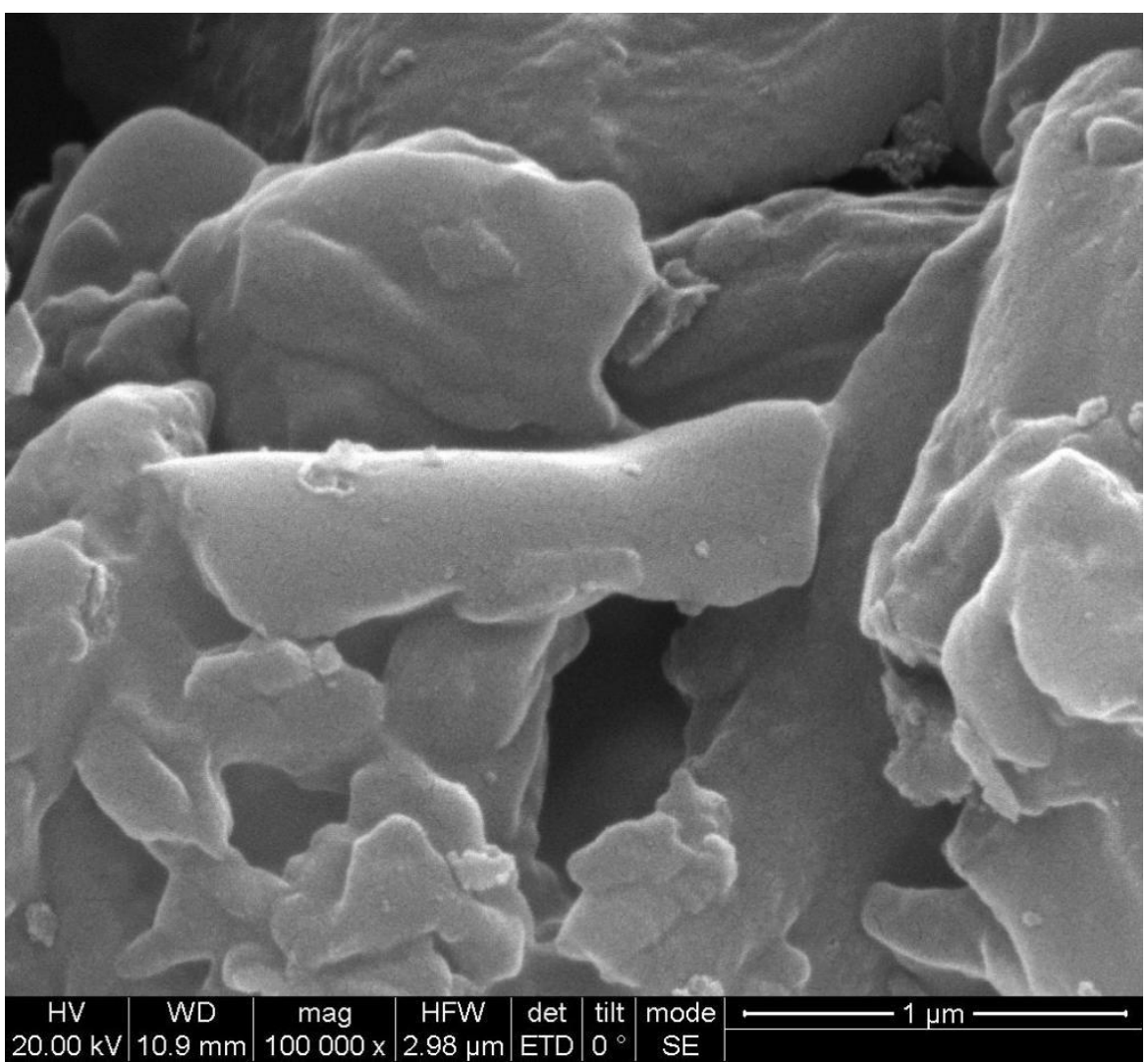

Figure S2. SEM of Co-CGBC 


\section{$5 \mathrm{~nm}$}

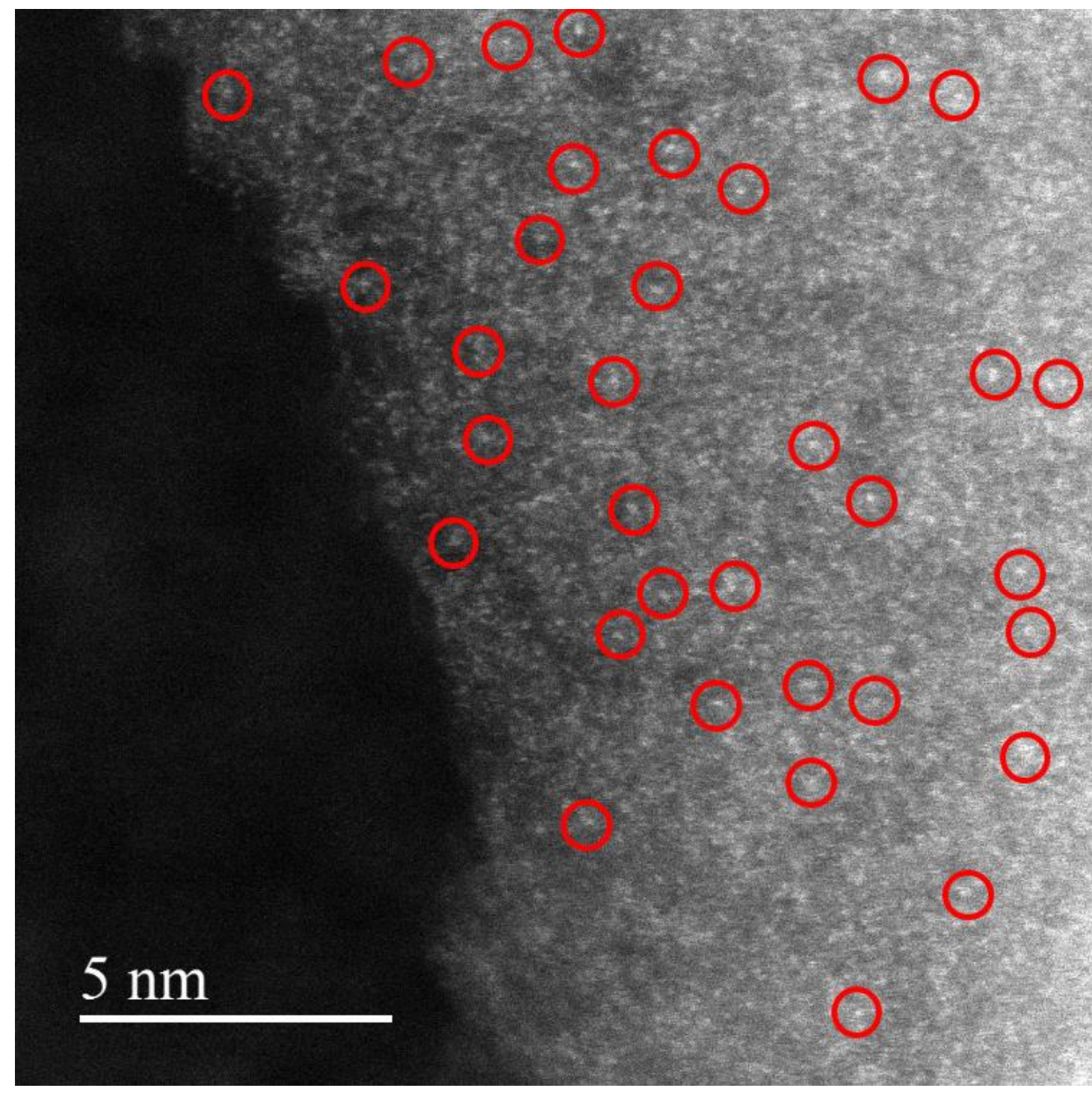




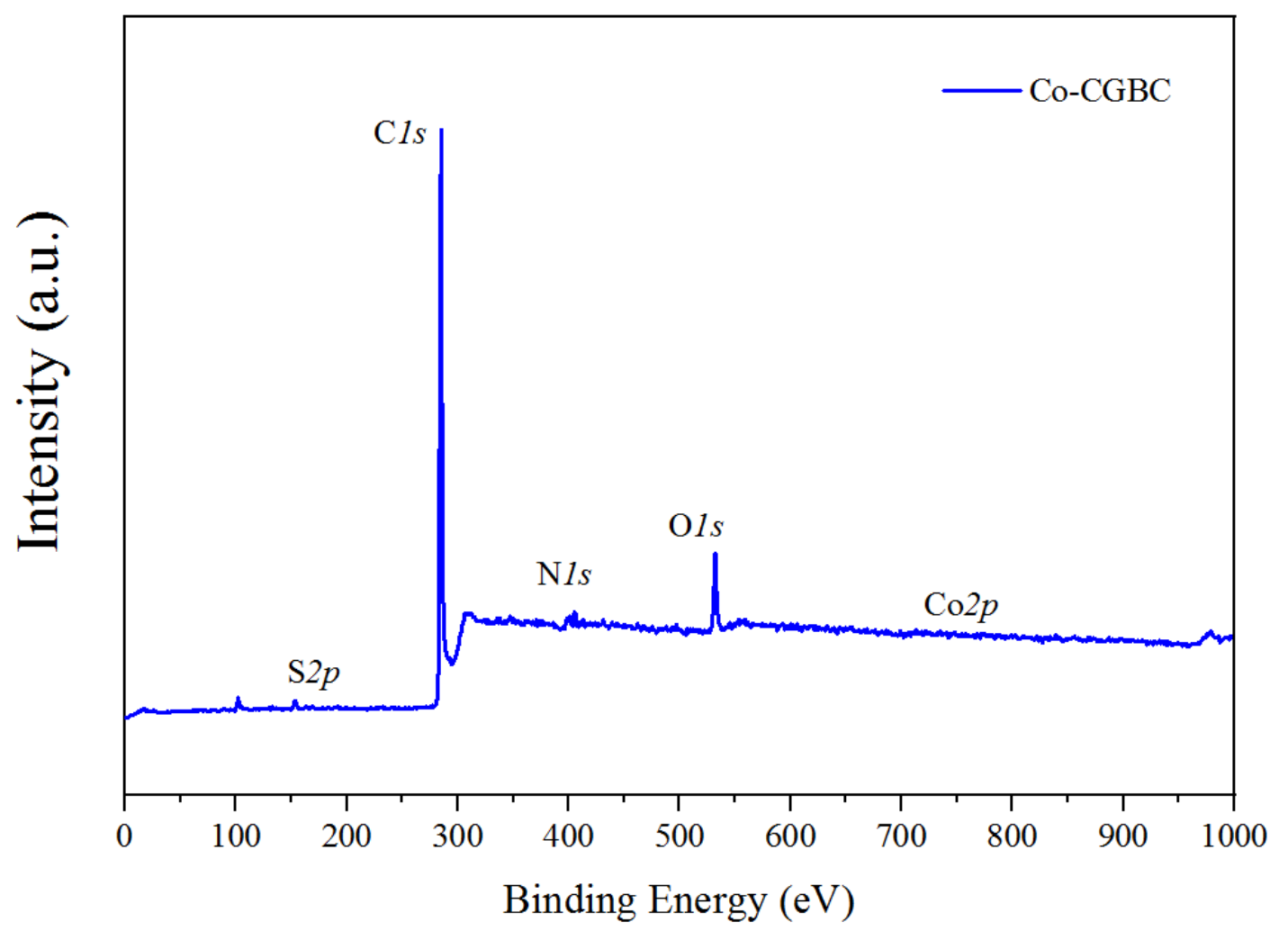

Figure S4. XPS spectra of Co-CGBC 

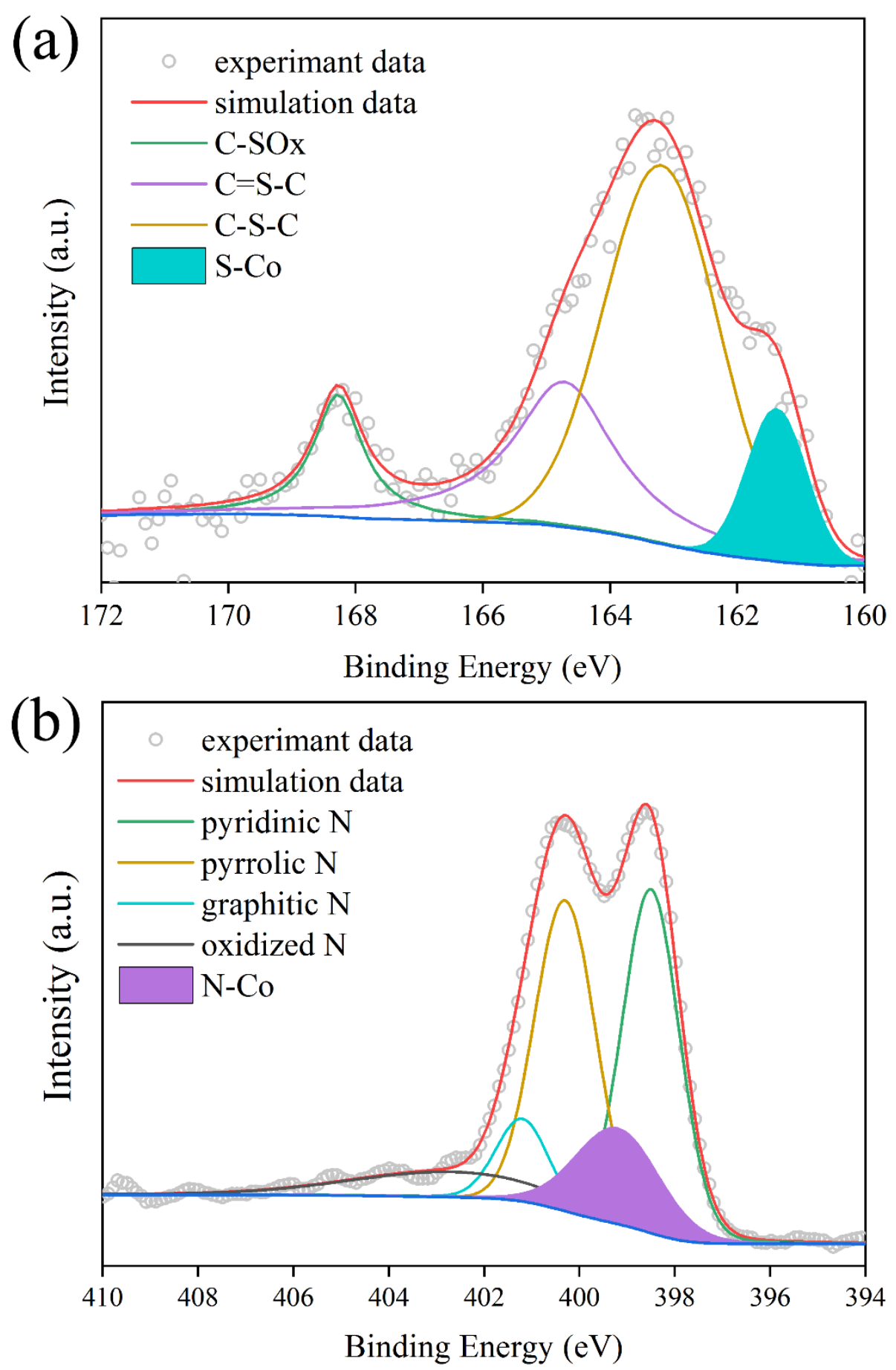

Figure S5. High-resolution S 2p (a) and N 1s (b) XPS spectra of Co-CGBC 


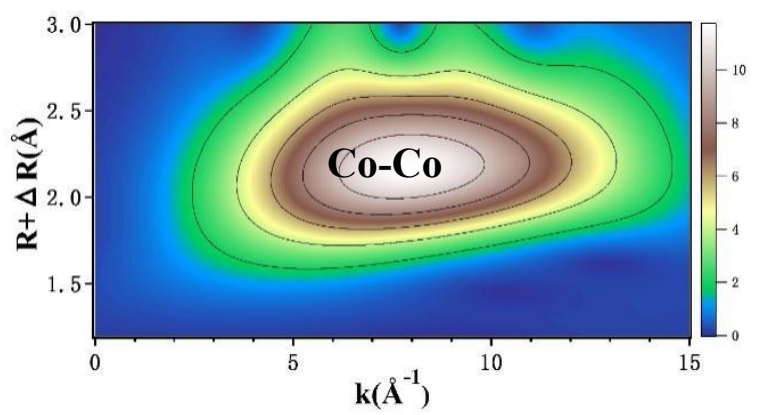

99

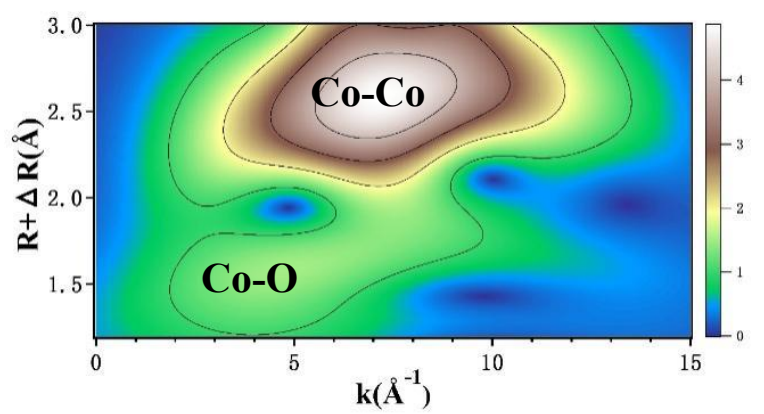

100
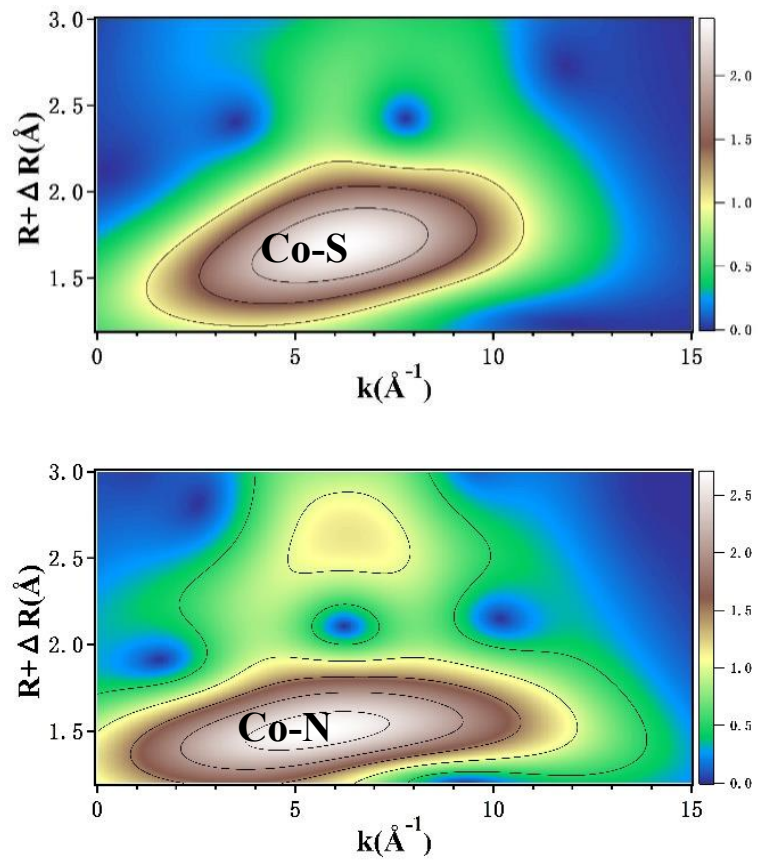

102 Figure S6. Wavelet transformed Co K-edge EXAFS of Co foil, CoO, CoS and CoPc 


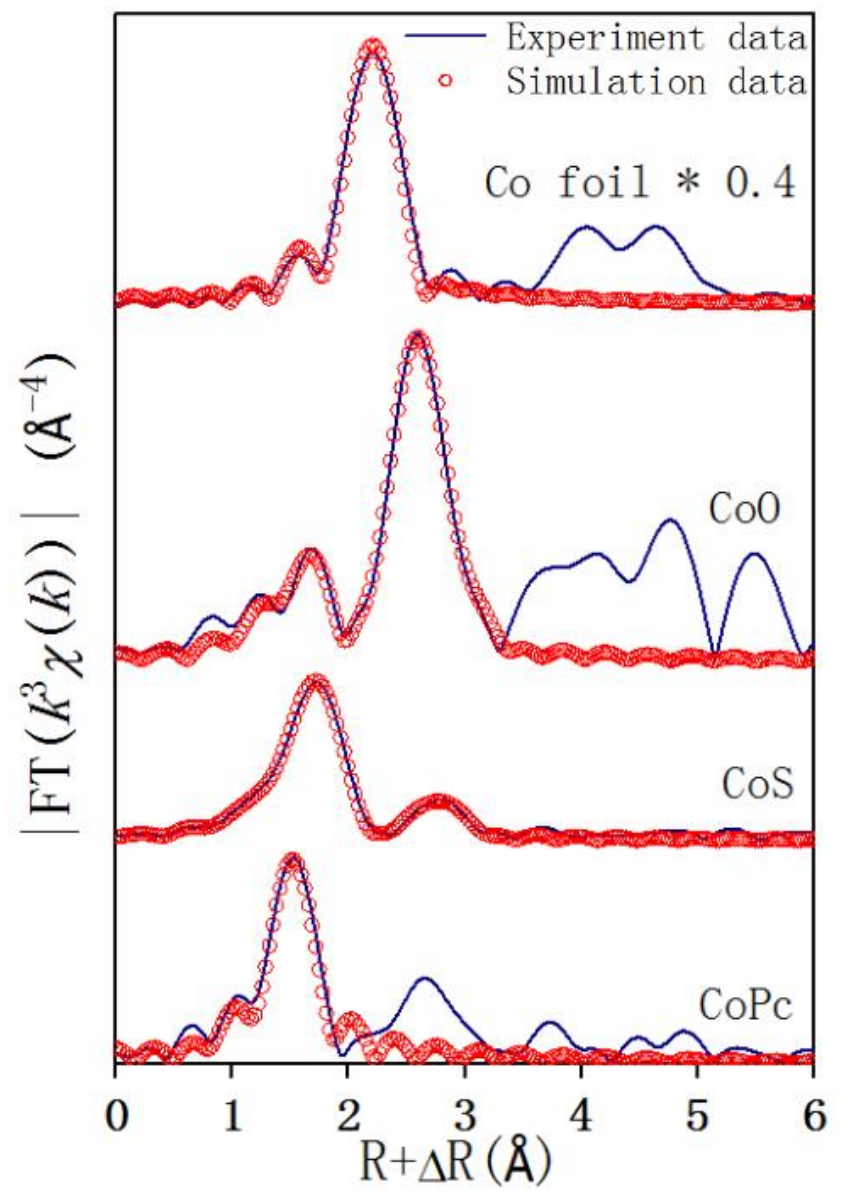

106 Figure S7. Shell-by-shell fitting of $k^{3}$-weighted Fourier transformed Co K-edge EXAFS 
(a)

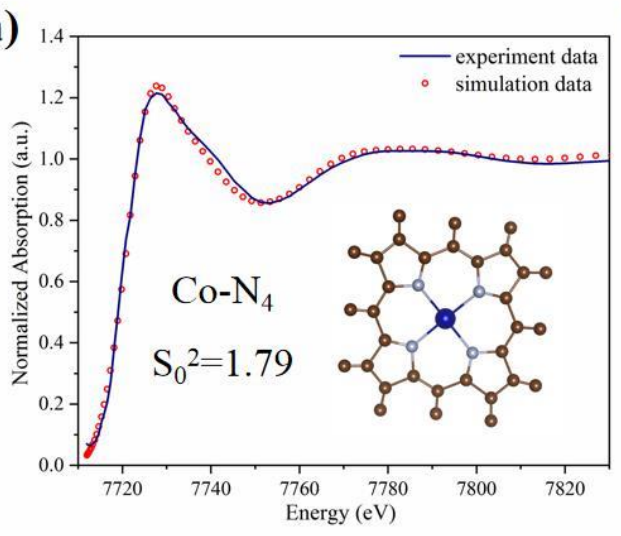

(c)

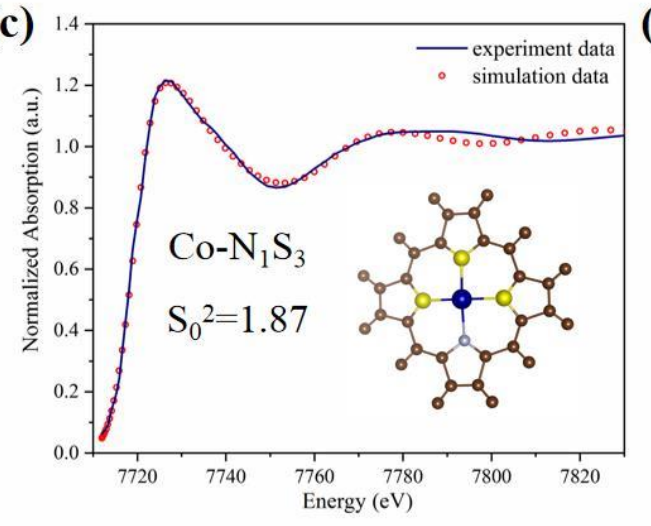

(b)

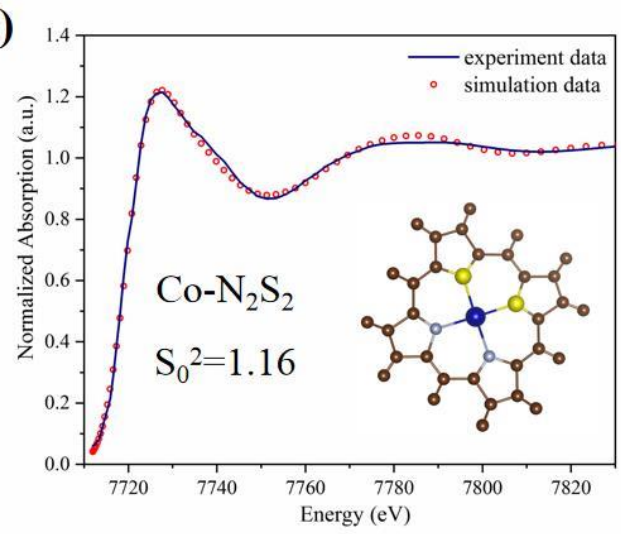

(d)

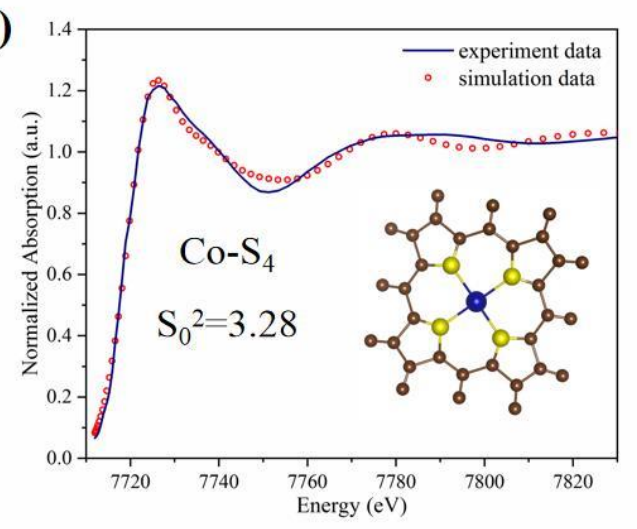

Figure S8. The Co K-edge XANES calculations of different Co-coordination structures: Co- $\mathrm{N}_{4}$

111 (a), Co- $\mathrm{N}_{2} \mathrm{~S}_{2}$ (b), Co-N $\mathrm{N}_{1} \mathrm{~S}_{3}$ (c) and $\mathrm{Co}-\mathrm{S}_{4}(\mathrm{~d})$.

112 
113

114

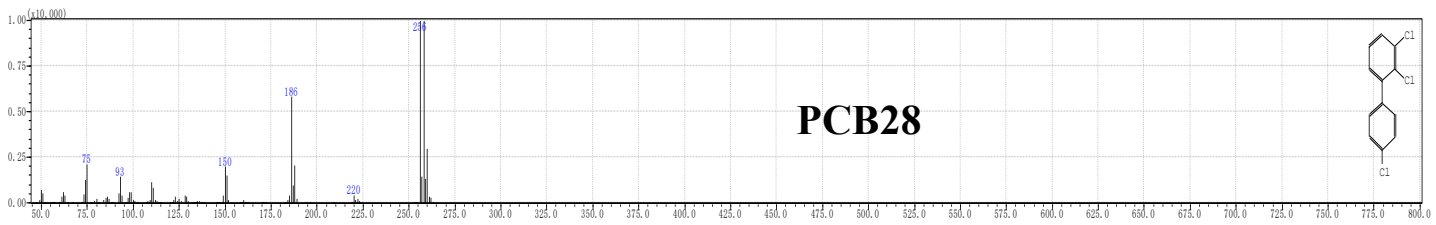

115

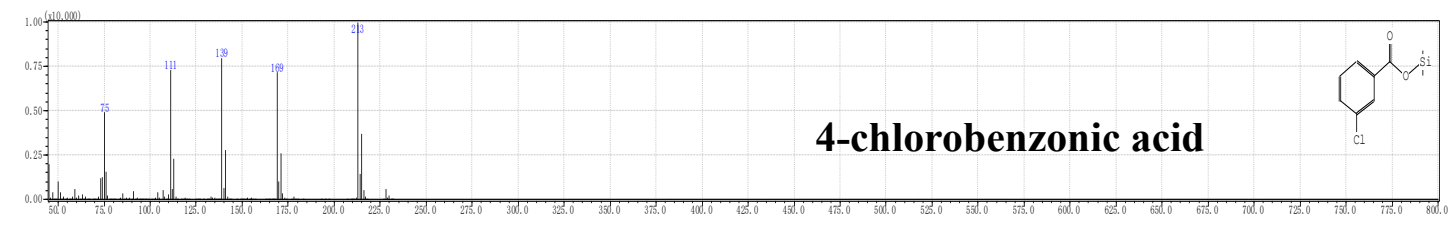

116

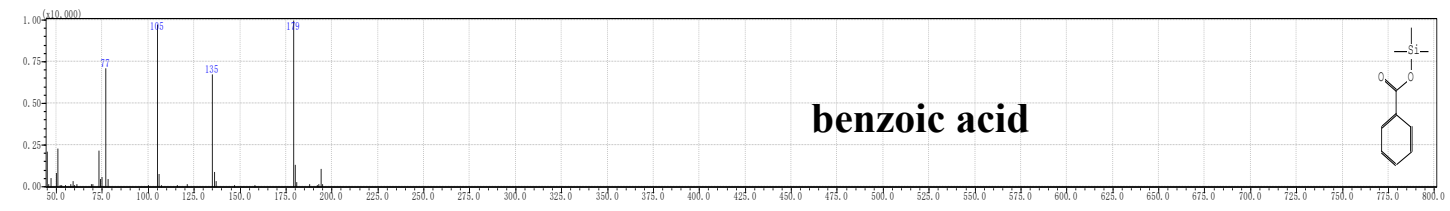

117

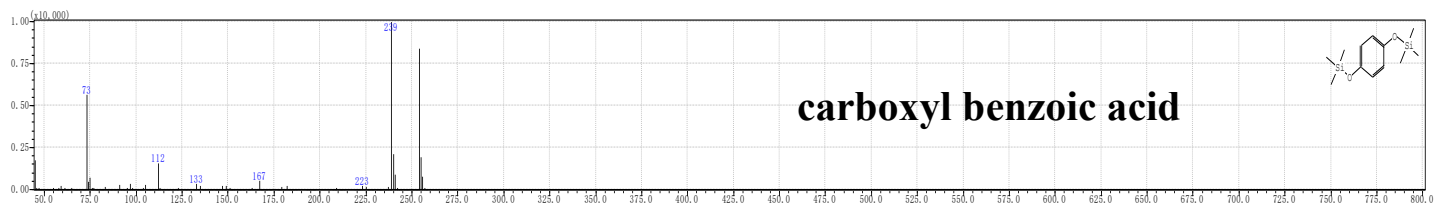

118

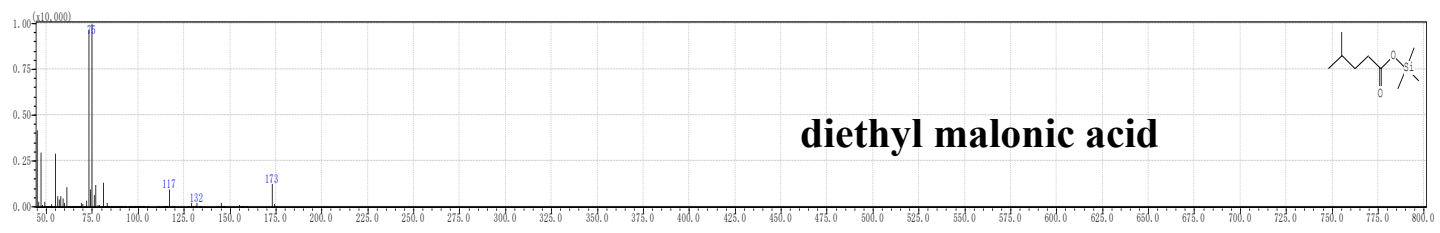

119

oxalic acid

120

Figure S9. The degradation intermediates of PCB28 identified by GC-MS

121 


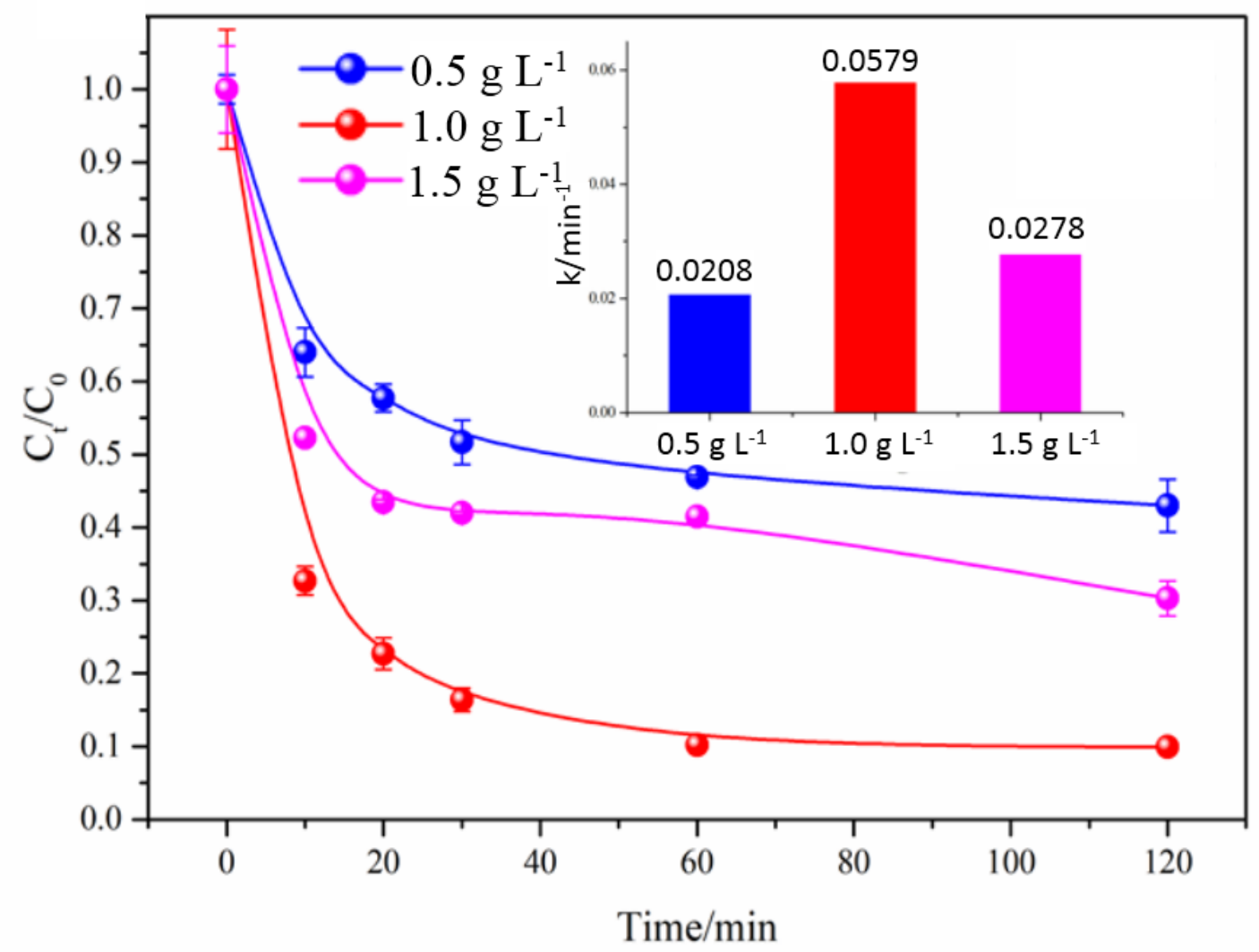

122

Figure S10. Effect of different catalyst dosages on PCB28 degradation

124 


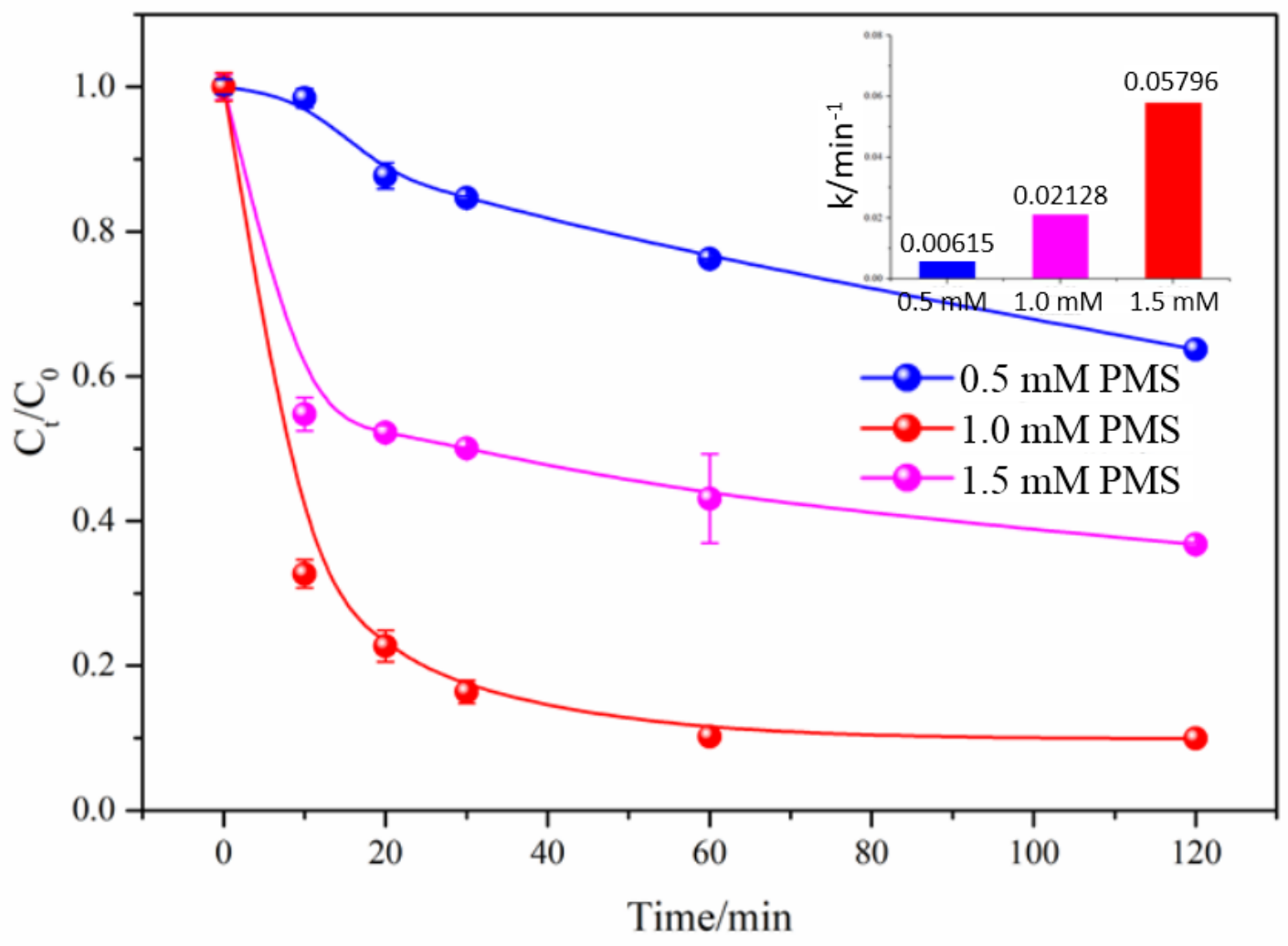

125

Figure S11. Effect of different PMS dosages on PCB28 degradation

127 


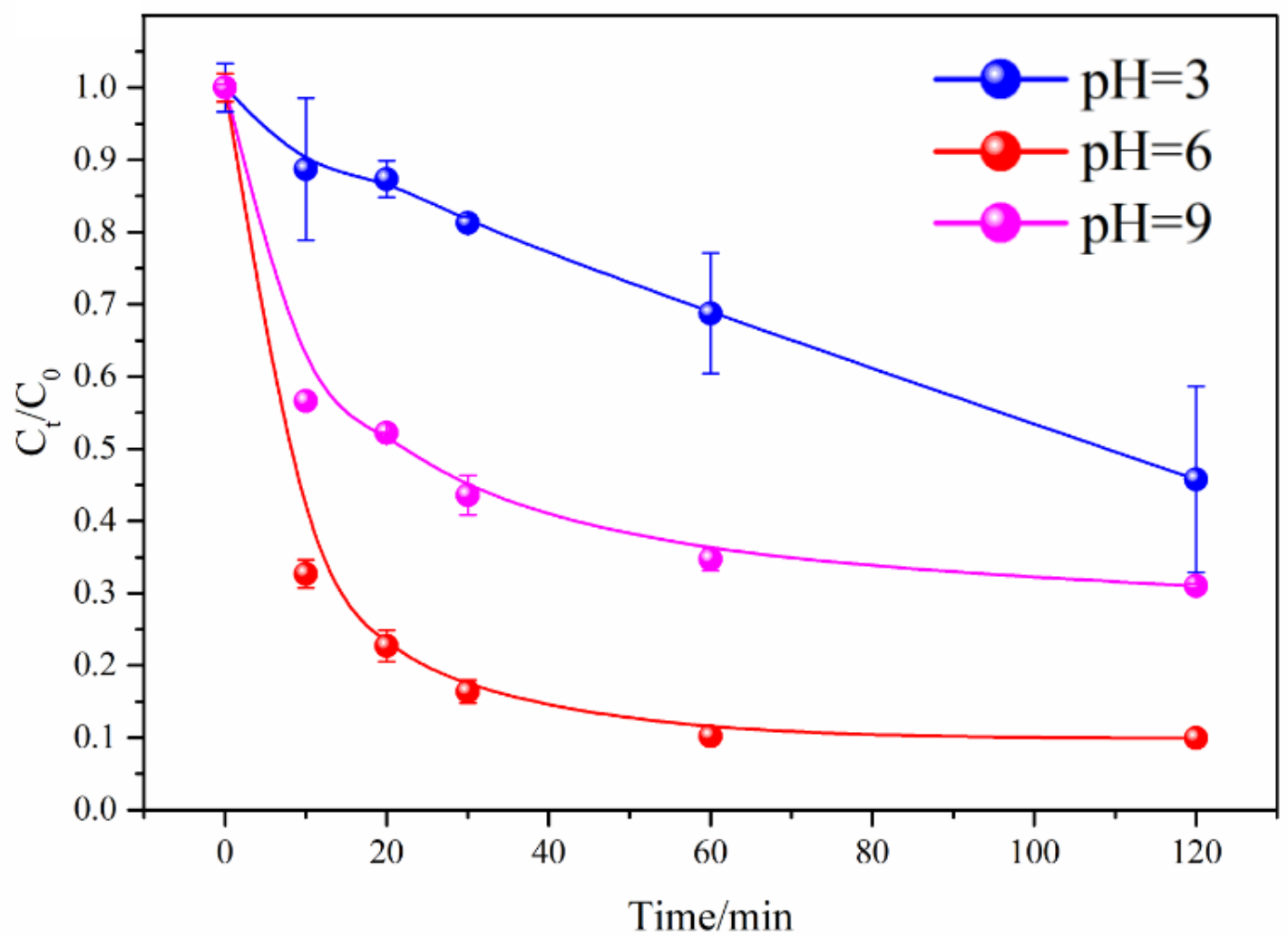

128

Figure S12. Effect of different $\mathrm{pH}$ on PCB28 degradation

130 


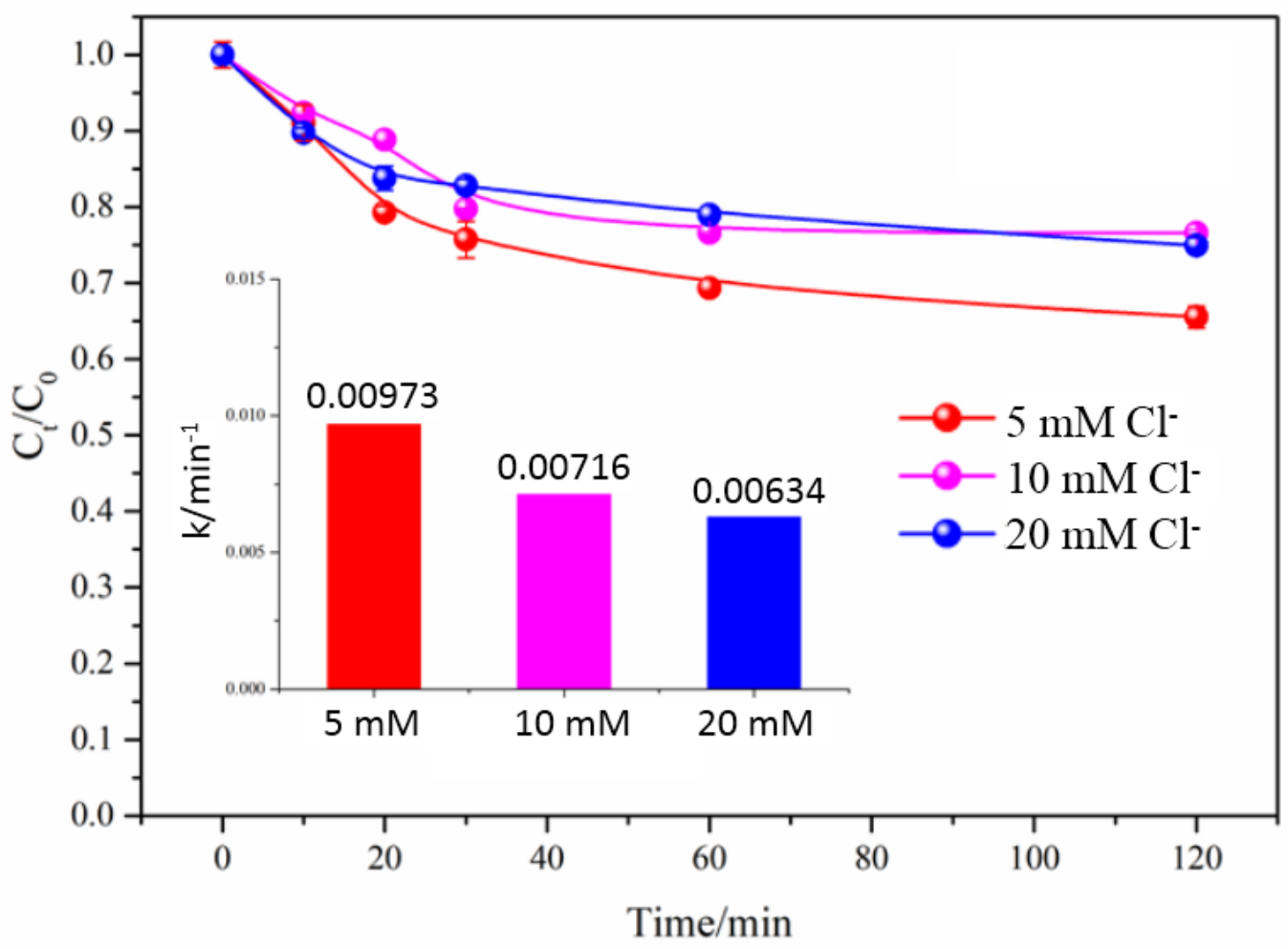

131

Figure S13. Effect of different $\mathrm{Cl}^{-}$dosages on PCB28 degradation 133 


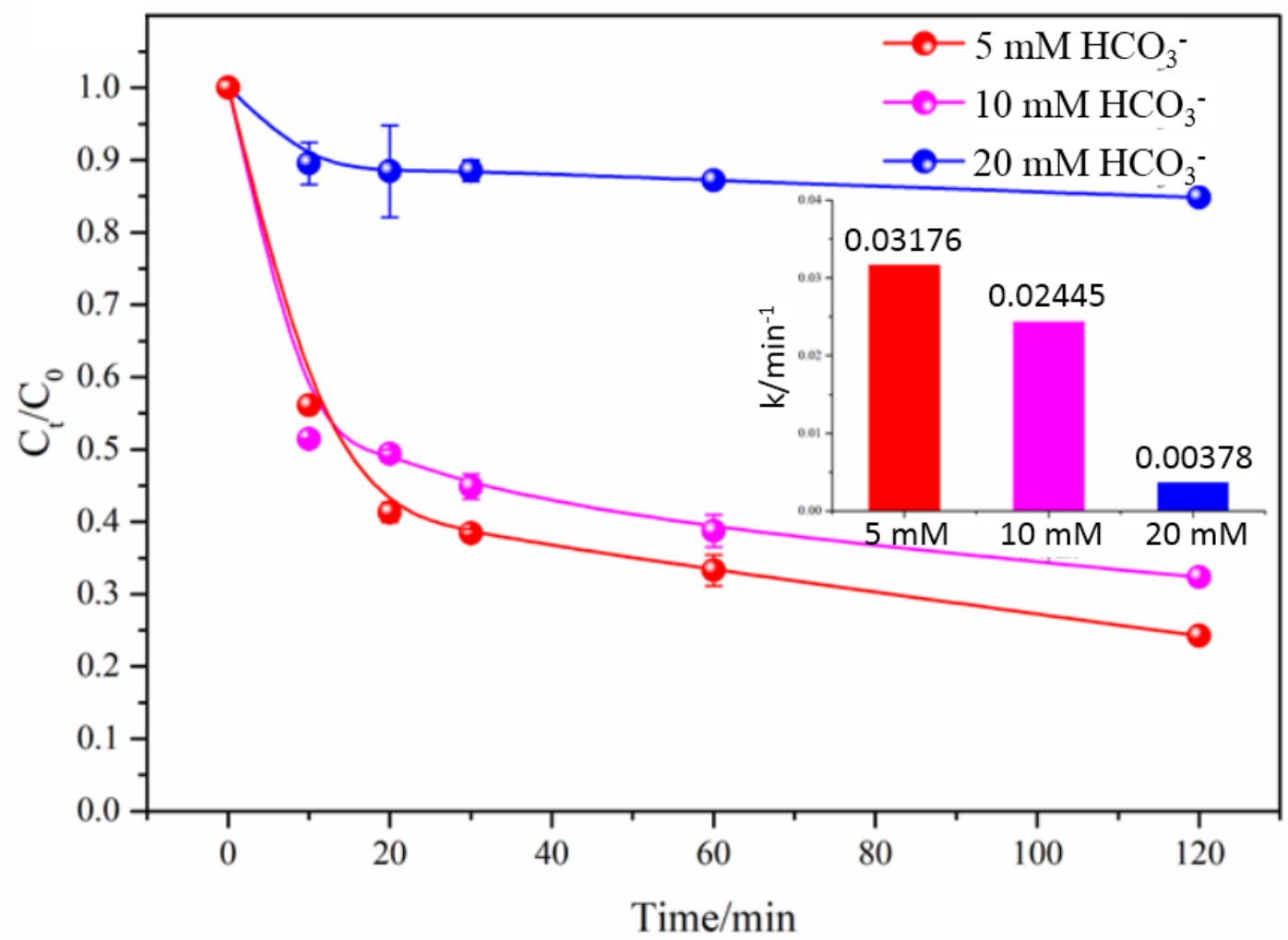

134

Figure S14. Effect of different $\mathrm{HCO}_{3}{ }^{-}$dosages on PCB28 degradation 136 


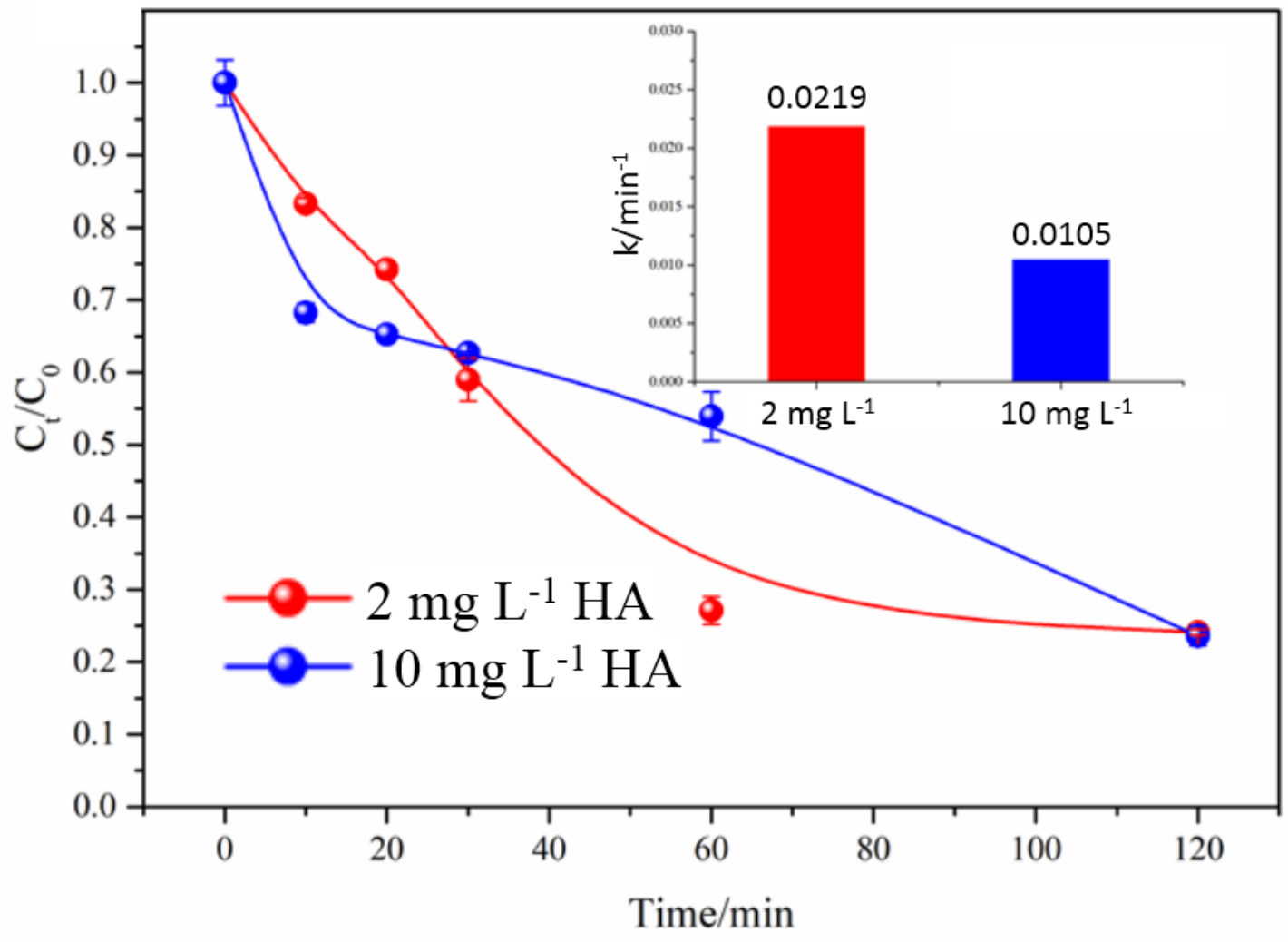

137

Figure S15. Effect of different HA dosages on PCB28 degradation

139 


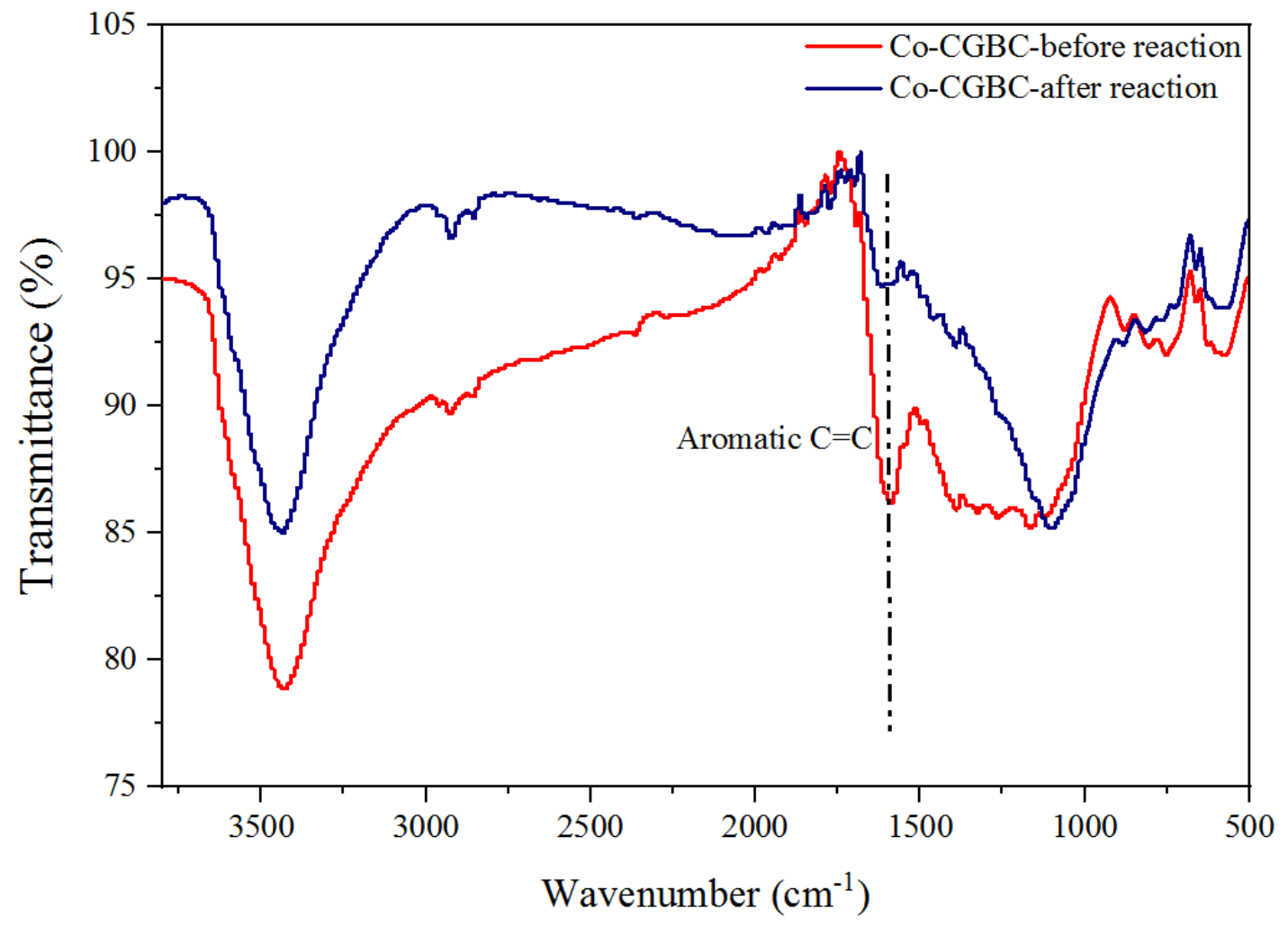

Figure S16. FT-IR of Co-CGBC before and after activation reaction 


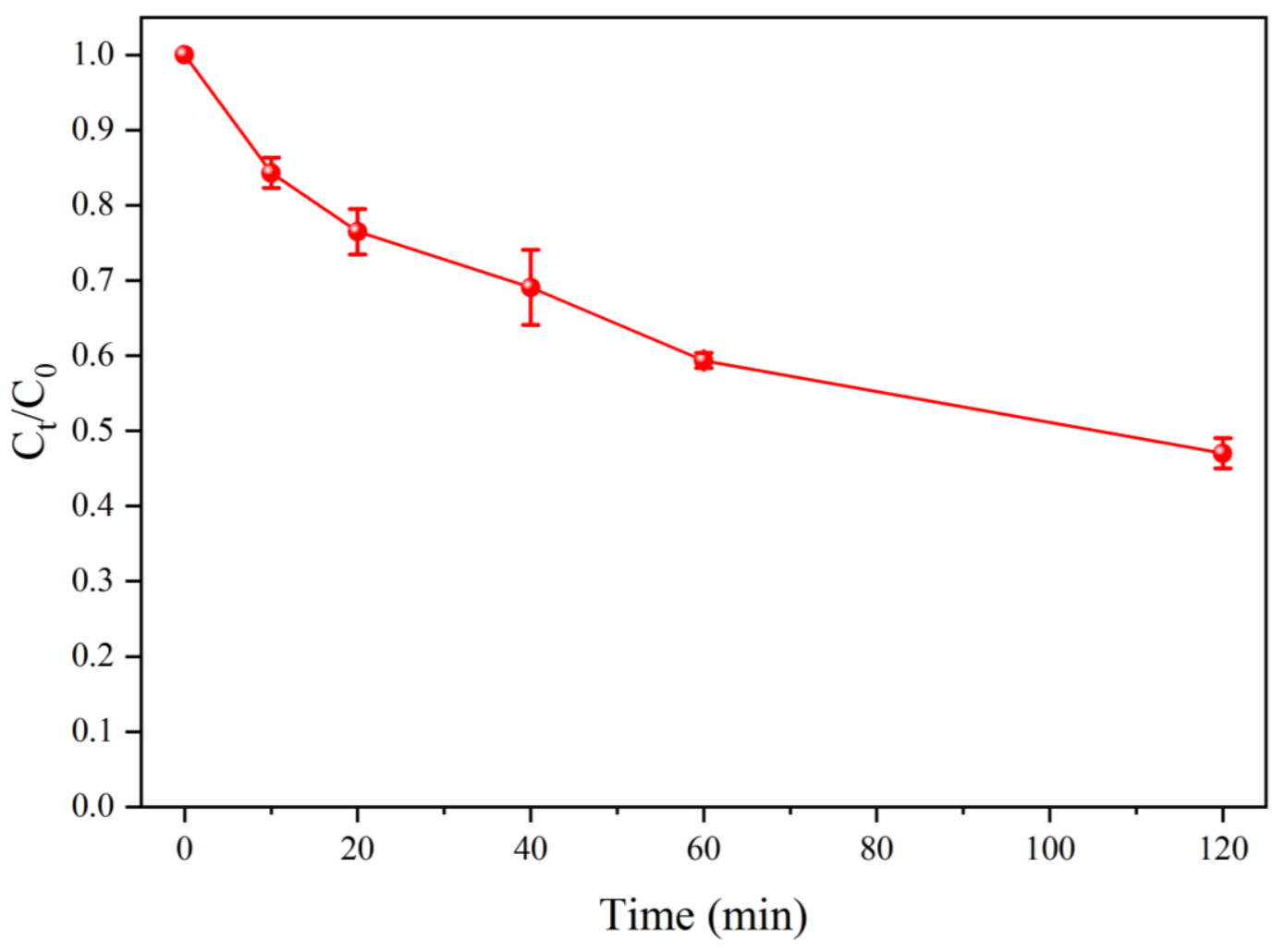

143

144

Figure S17. PCB degradation in the actual water from Xuanwu Lake

145 


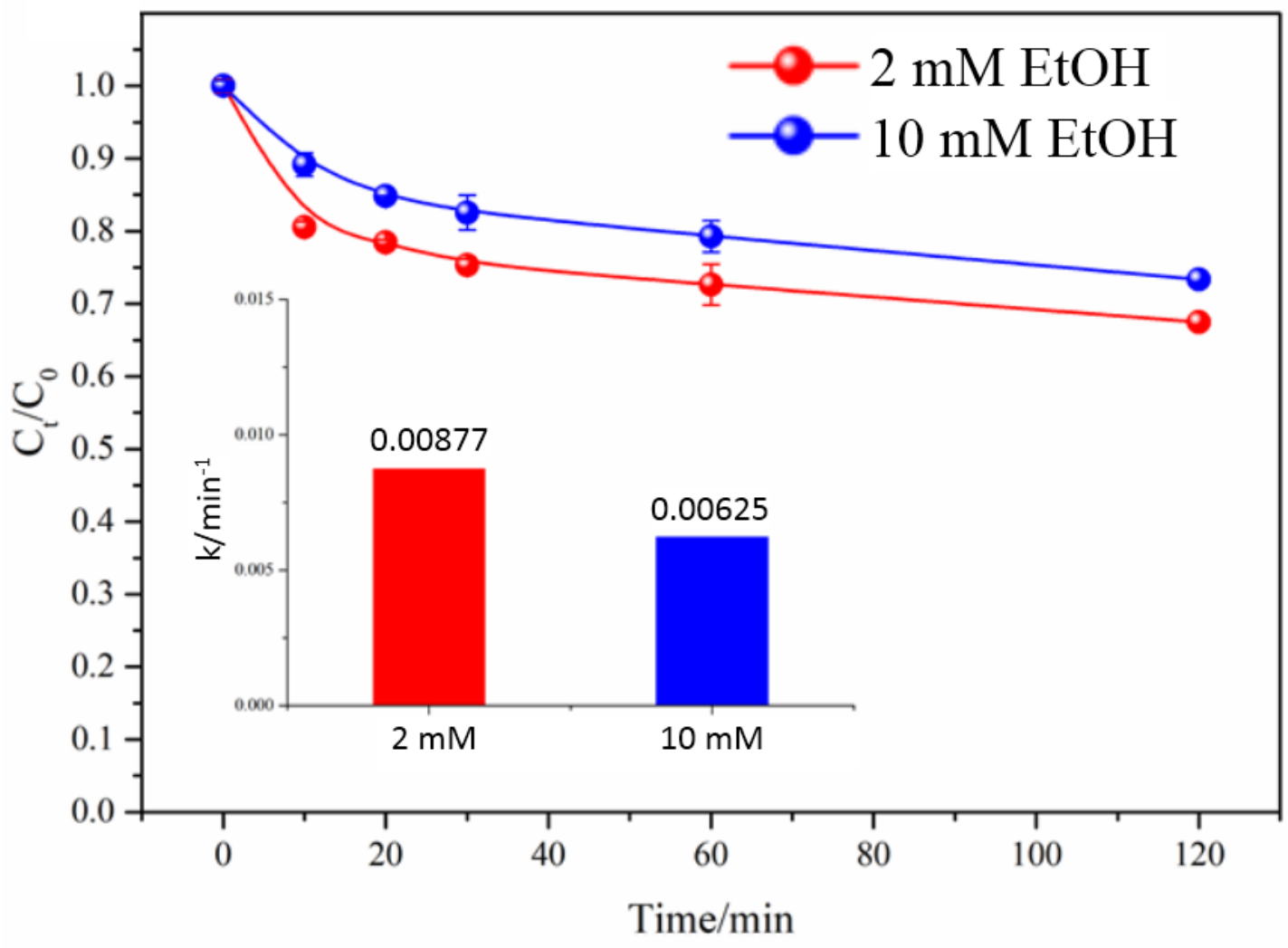

146

Figure S18. Effect of different EtOH dosages on PCB28 degradation 148 


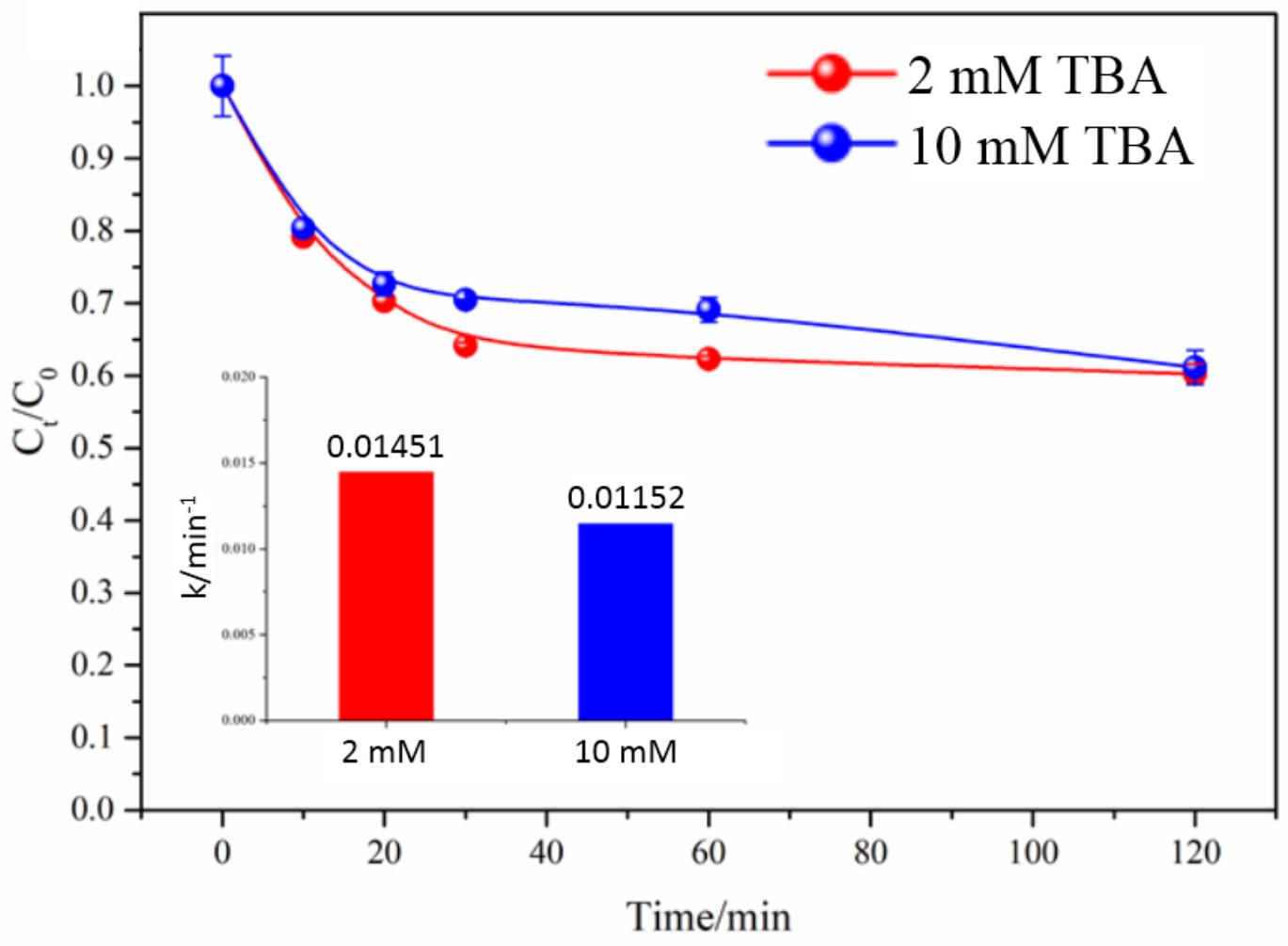

150

Figure S19. Effect of TBA scavenger dosages on PCB28 degradation 151 


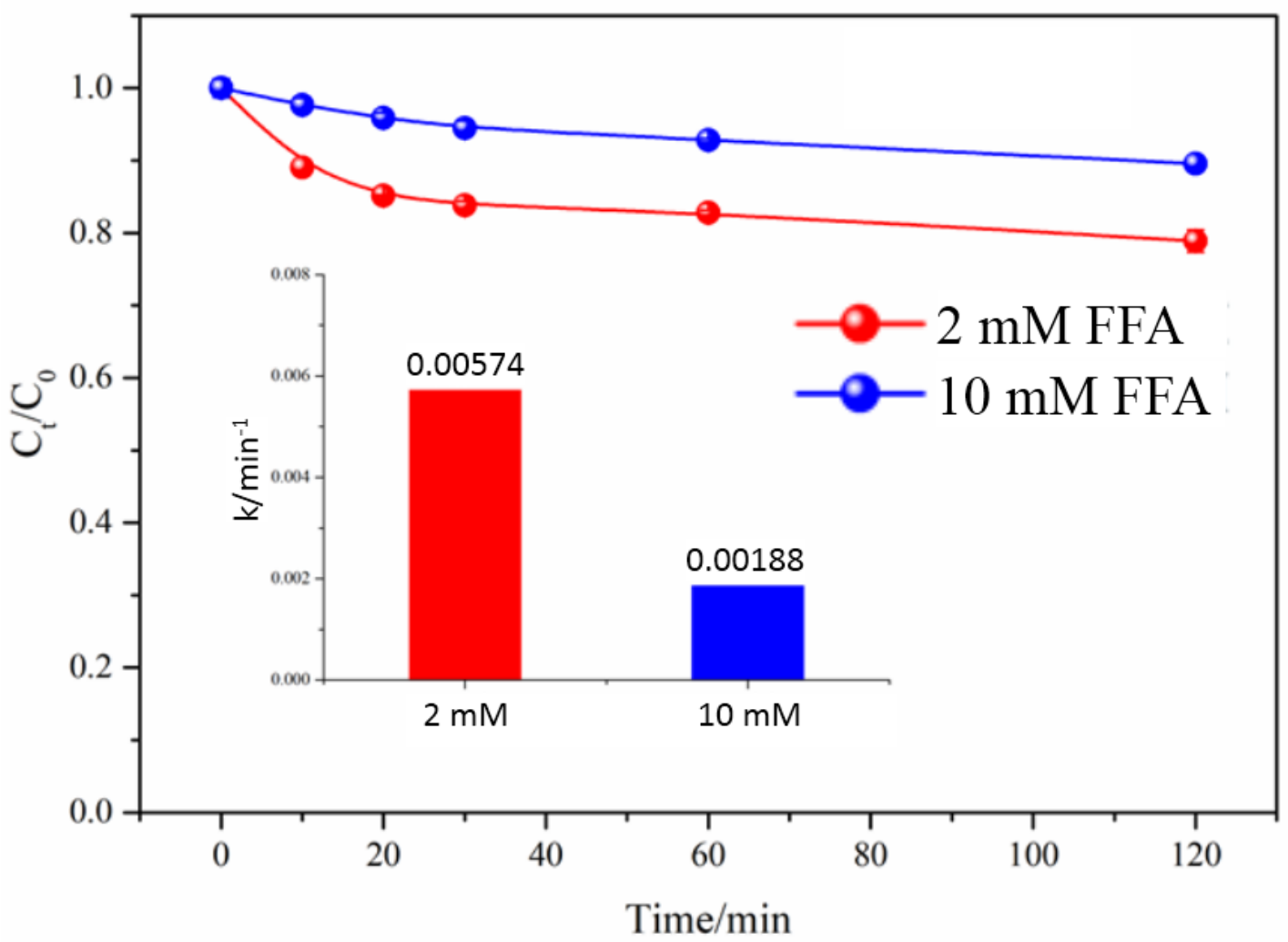

152

Figure S20. Effect of FFA scavenger dosages on PCB28 degradation 154 


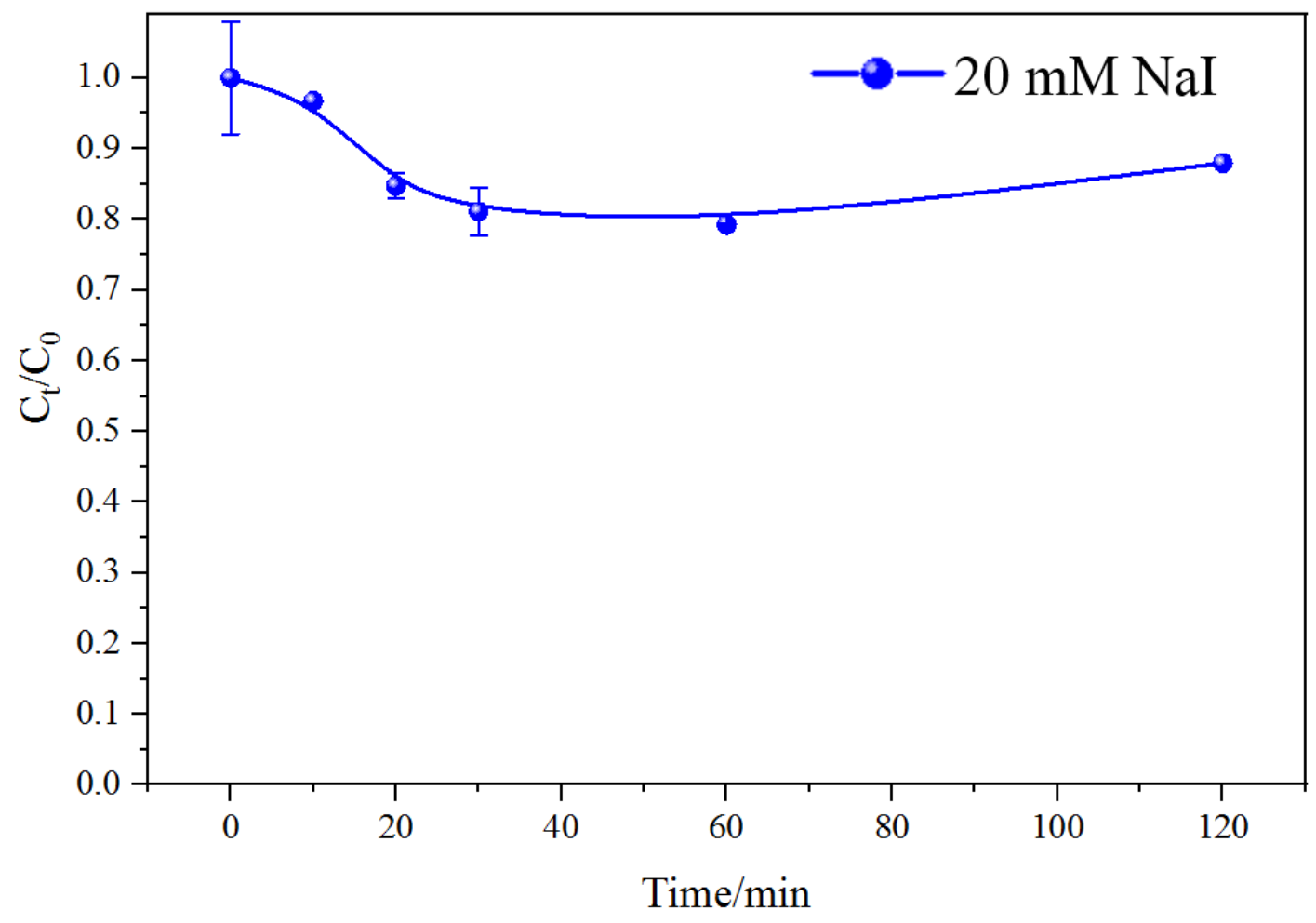

155

Figure S21. Effect of NaI on PCB28 degradation

157 


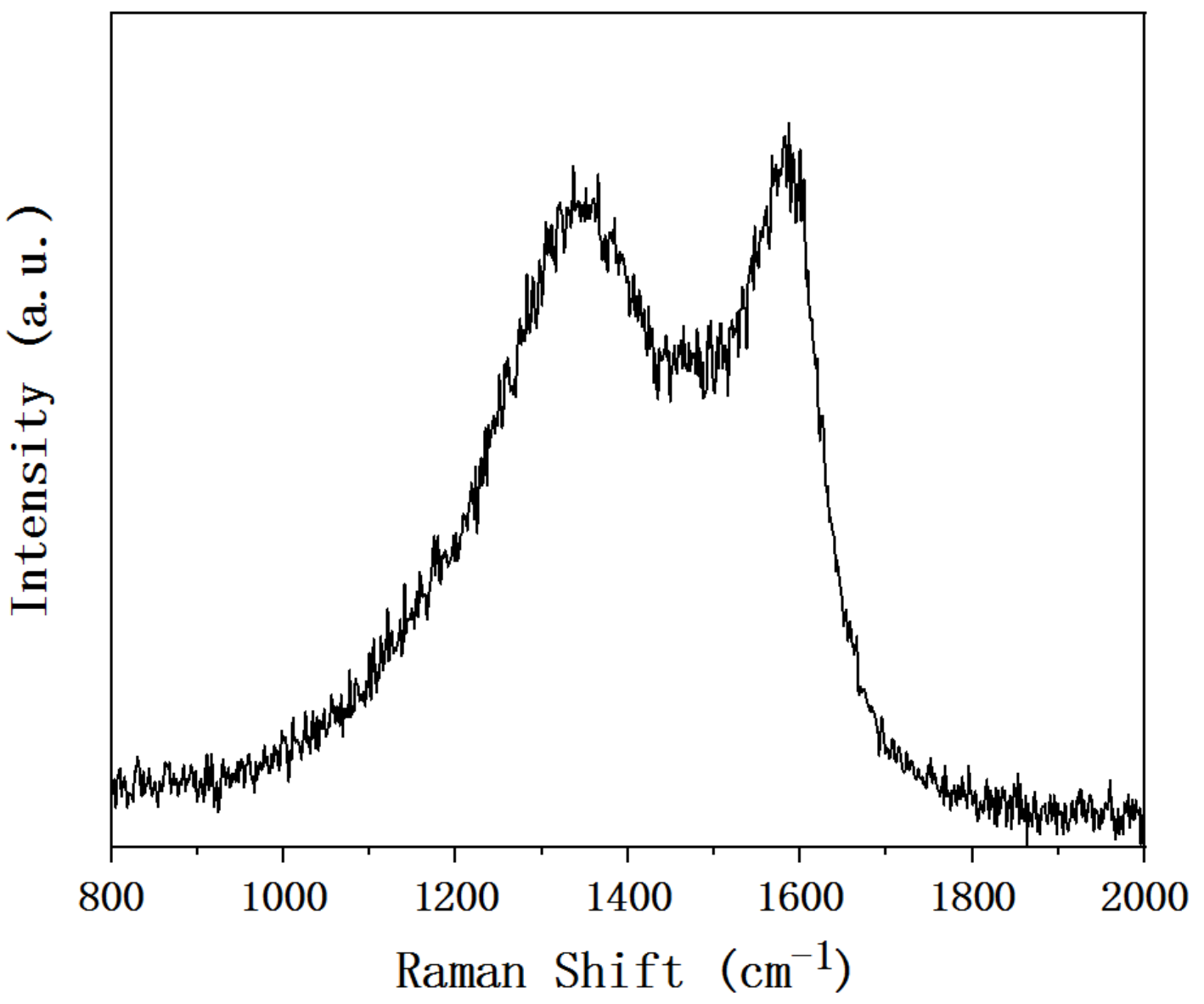

159

Figure S22. Raman spectrum of Co-CGBC

160 


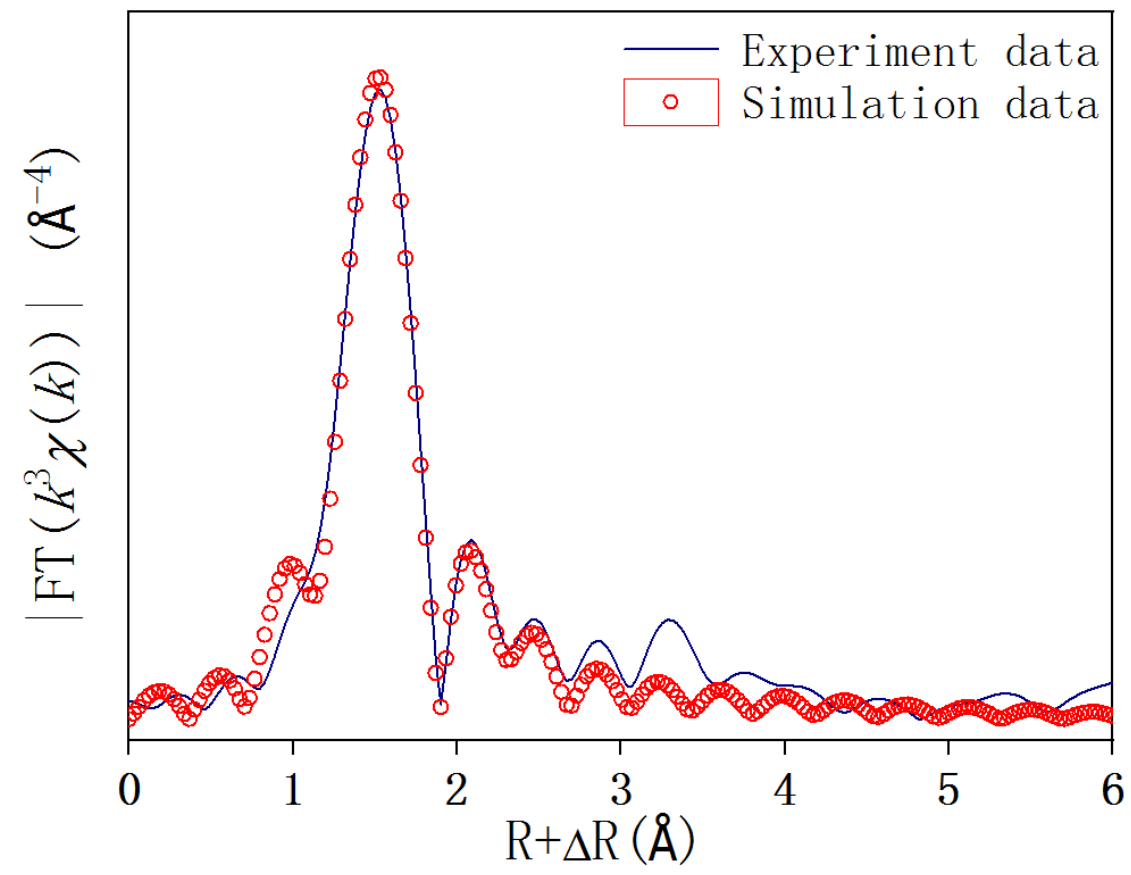

162 Figure S23. Shell-by-shell fitting of $\mathrm{k}^{3}$-weighted Fourier transformed Co K-edge EXAFS 163 of Co-CGBC after activation reaction 


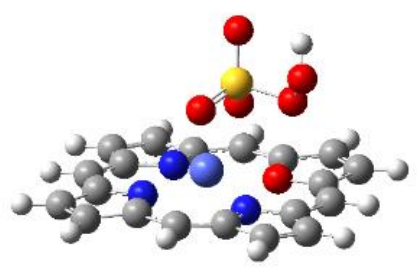

$\mathrm{Co}-\mathrm{N}_{3} \mathrm{O} / \mathrm{PMS}$

$-4.73 \mathrm{eV}$

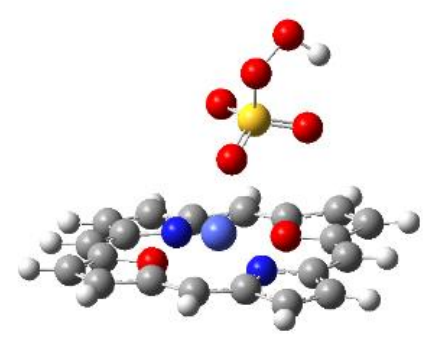

$\mathrm{CO}-\mathrm{N}_{2} \mathrm{O}_{2} / \mathrm{PMS}$

$-1.57 \mathrm{eV}$

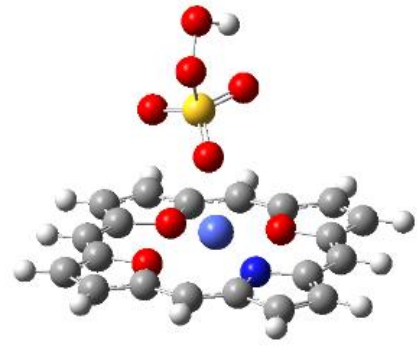

$\mathrm{Co}-\mathrm{NO}_{3} / \mathrm{PMS}$

$1.30 \mathrm{eV}$

Figure S24. The binding energies calculated by DFT with different coordination structures

around Co atom 

Table S1 The basic properties of spent coffee ground and Co-CGBC

\begin{tabular}{ccccccc}
\hline & C[\%] & N[\%] & H[\%] & S[\%] & Co[\%] & BET $\left(\mathbf{m}^{\mathbf{2}} \mathbf{g}^{-1}\right)$ \\
\hline Spent coffee ground 1 & 52.05 & 2.35 & 6.52 & 0.55 & $\backslash$ & 1.9524 \\
Spent coffee ground 2 & 49.92 & 2.15 & 7.35 & 0.54 & $\backslash$ & 1.7907 \\
Co-CGBC 1 & 59.97 & 14.80 & 2.73 & 1.42 & 1.13 & 293.9942 \\
Co-CGBC 2 & 59.91 & 14.85 & 2.80 & 1.50 & 1.15 & 275.2818 \\
\hline
\end{tabular}

170

171

172

173 
174 Table S2. EXAFS fitting parameters at the Co K-edge for various samples $\left(S_{0}^{2}=0.776\right)$

\begin{tabular}{|c|c|c|c|c|c|c|}
\hline Sample & Shell & $N^{a}$ & $R\left(\AA{ }^{b}\right)^{b}$ & $\sigma^{2}\left(\AA^{2}\right)^{c}$ & $\Delta E_{0}(\mathrm{eV})^{d}$ & $R$ factor \\
\hline Co foil & Co-Co & 12 & 2.49 & 0.0062 & 7.1 & 0.0004 \\
\hline \multirow{3}{*}{$\mathrm{CoO}$} & $\mathrm{Co}-\mathrm{O}$ & 6.0 & 2.06 & 0.0101 & \multirow{3}{*}{-3.6} & \multirow{3}{*}{0.0004} \\
\hline & $\mathrm{Co}-\mathrm{Co}$ & 12.5 & 2.99 & 0.0095 & & \\
\hline & $\mathrm{Co}-\mathrm{O}$ & 6.2 & 3.66 & 0.0101 & & \\
\hline \multirow{2}{*}{$\mathrm{CoS}$} & Co-S & 6.0 & 2.21 & 0.0089 & \multirow{2}{*}{-9.1} & \multirow{2}{*}{0.0001} \\
\hline & $\mathrm{Co}-\mathrm{Co}$ & 6.0 & 3.28 & 0.0114 & & \\
\hline $\mathrm{CoPc}$ & Co-N & 4.0 & 1.91 & 0.0016 & 6.8 & 0.0025 \\
\hline \multirow{2}{*}{$\begin{array}{c}\text { Co-CGBC } \\
\text { before } \\
\text { reaction }\end{array}$} & Co-N & 2.8 & 2.01 & 0.0058 & \multirow{2}{*}{-0.3} & \multirow{2}{*}{0.0006} \\
\hline & Co-S & 1.2 & 2.19 & 0.0102 & & \\
\hline \multirow{2}{*}{$\begin{array}{l}\text { Co-CGBC } \\
\text { after } \\
\text { reaction }\end{array}$} & Co-N & 2.9 & 2.01 & 0.0047 & \multirow{2}{*}{4.2} & \multirow{2}{*}{0.0005} \\
\hline & Co-S & 1.7 & 2.20 & 0.0113 & & \\
\hline
\end{tabular}

$175{ }^{a} N$ : coordination numbers; ${ }^{b} R$ : bond distance; ${ }^{c} \sigma^{2}$ : Debye-Waller factors; ${ }^{d} \Delta E_{0}$ : the inner potential

176 correction. $R$ factor: goodness of fit. $S_{0}{ }^{2}$ was set to 0.776 , according to the experimental EXAFS

177 fit of Co foil reference by fixing $\mathrm{CN}$ as the known crystallographic value. 
Table S3 The basic properties of the actual water from Xuanwu Lake

\begin{tabular}{ccccccc}
\hline & $\mathrm{pH}$ & $\begin{array}{c}\text { Conductivity } \\
\mu \mathrm{s} \mathrm{cm}^{-1}\end{array}$ & $\begin{array}{c}\mathrm{TOC} \\
\mathrm{mg} \mathrm{L}^{-1}\end{array}$ & $\begin{array}{c}\mathrm{HCO}_{3}^{-} \\
\mathrm{mg} \mathrm{L}^{-1}\end{array}$ & $\begin{array}{c}\mathrm{Cl}^{-} \\
\mathrm{mg} \mathrm{L}^{-1}\end{array}$ & $\begin{array}{c}\mathrm{NO}_{3}^{-} \\
\mathrm{mg} \mathrm{L}^{-1}\end{array}$ \\
\hline actual water & 6.24 & 176.8 & 25.8 & 17.5 & 14.2 & 4.33 \\
\hline
\end{tabular}

180

181 
Table S4. Comparison of other Co-based catalysts for AOPs and pollutant degradation

\begin{tabular}{|c|c|c|c|c|c|c|c|c|}
\hline $\begin{array}{c}\text { Referenc } \\
\text { e }\end{array}$ & Catalyst & $\begin{array}{l}\text { Catalyst } \\
\text { Dosage }\end{array}$ & Co Dosage & Oxidant & Pollutant & $\begin{array}{c}\text { Degradation } \\
\text { Efficiency }\end{array}$ & $\mathrm{K}_{\mathrm{obs}}\left(\min ^{-1}\right)$ & $\begin{array}{c}\text { Metal leaching } \\
\text { initial } \mathrm{pH}\end{array}$ \\
\hline$[5]$ & CoMgAI-LDH & $0.84 \mathrm{~g} \mathrm{~L}^{-1}$ & $17.7 \mathrm{mg} \mathrm{L}^{-1}$ & $\begin{array}{c}\mathrm{H}_{2} \mathrm{O}_{2} \\
50 \mathrm{mM}\end{array}$ & MO $100 \mathrm{mg} \mathrm{L}^{-1}$ & 100\% $60 \mathrm{~min}$ & No data & $\begin{array}{c}\text { No leaching } \\
\mathrm{pH}=8.3\end{array}$ \\
\hline [6] & CoCuAl-LDH & $0.1 \mathrm{~g} \mathrm{~L}^{-1}$ & $23.7 \mathrm{mg} \mathrm{L}^{-1}$ & $\begin{array}{c}\text { PMS } \\
0.33 \mathrm{mM}\end{array}$ & $\mathrm{AO} 720 \mathrm{mg} \mathrm{L}^{-1}$ & $100 \% 30 \mathrm{~min}$ & $0.057 \mathrm{~min}^{-1}$ & $\begin{array}{c}\text { Co } 0.06 \mathrm{mg} \mathrm{L}^{-1} \\
\text { Cu } 0.37 \mathrm{mg} \mathrm{L}^{-1} \\
\mathrm{pH}=6.3\end{array}$ \\
\hline [7] & CoMgAl-LDH & $75 \mathrm{mg} \mathrm{L}^{-1}$ & $16.8 \mathrm{mg} \mathrm{L}^{-1}$ & $\begin{array}{c}\text { PMS } \\
0.4 \mathrm{mM}\end{array}$ & ATZ $10 \mathrm{mg} \mathrm{L}^{-1}$ & $95 \% 15$ min & $0.239 \mathrm{~min}^{-1}$ & $\begin{array}{c}\text { Co } 0.15 \mathrm{mg} \mathrm{L}^{-1} \\
\mathrm{pH}=6.3\end{array}$ \\
\hline [8] & $\begin{array}{c}\text { CuOx@Co- } \\
\text { LDH }\end{array}$ & $0.3 \mathrm{~g} \mathrm{~L}^{-1}$ & unknown & $\begin{array}{c}\text { PS } \\
5 \mathrm{mM}\end{array}$ & Phenol $25 \mathrm{mg} \mathrm{L}^{-1}$ & 100\% $30 \mathrm{~min}$ & $0.170 \mathrm{~min}^{-1}$ & $\begin{array}{c}\text { No Co leaching } \\
\text { Cu } 0.08 \mathrm{mg} \mathrm{L}^{-1} \\
\mathrm{pH}=6.5\end{array}$ \\
\hline [9] & CoMn-LDH & $25 \mathrm{mg} \mathrm{L}^{-1}$ & $10.3 \mathrm{mg} \mathrm{L}^{-1}$ & $\begin{array}{c}\text { PMS } \\
0.33 \mathrm{mM}\end{array}$ & Orange $\mathrm{G} 50 \mathrm{mg} \mathrm{L}^{-1}$ & $100 \% 4$ min & No data & $\begin{array}{c}\mathrm{Mn} 0.16 \mathrm{mg} \mathrm{L}^{-1} \\
\text { Co } 0.53 \mathrm{mg} \mathrm{L}^{-1} \\
\mathrm{pH}=6.78\end{array}$ \\
\hline [10] & FeCo@NC & $0.1 \mathrm{~g} \mathrm{~L}^{-1}$ & $13.5 \mathrm{mg} \mathrm{L}^{-1}$ & $\begin{array}{c}\text { PMS } \\
0.65 \mathrm{mM}\end{array}$ & BPA $20 \mathrm{mg} \mathrm{L}^{-1}$ & $100 \% 6$ min & $1.25 \mathrm{~min}^{-1}$ & $\begin{array}{c}\text { Co } 0.23 \mathrm{mg} \mathrm{L}^{-1} \\
\mathrm{pH}=6.0\end{array}$ \\
\hline [11] & Co-TPML & $0.2 \mathrm{~g} \mathrm{~L}^{-1}$ & $0.26 \mathrm{mg} \mathrm{L}^{-1}$ & $\begin{array}{l}\text { PMS } \\
2 \mathrm{mM}\end{array}$ & BPA $11.5 \mathrm{mg} \mathrm{L}^{-1}$ & $100 \% 5$ min & No data & $\begin{array}{c}\text { Co } 0.1 \mathrm{ug} \mathrm{L}^{-1} \\
\mathrm{pH}=3.0\end{array}$ \\
\hline This work & Co-CGBC & $1 \mathrm{~g} \mathrm{~L}^{-1}$ & $11.3 \mathrm{mg} \mathrm{L}^{-1}$ & $\begin{array}{c}\text { PMS } \\
2 \mathrm{mM}\end{array}$ & $\begin{array}{l}\text { PCB28 } 2 \mathrm{mg} \mathrm{L}^{-1} \\
\text { BPA } 20 \mathrm{mg} \mathrm{L}^{-1}\end{array}$ & $\begin{array}{l}90 \% 120 \mathrm{~min} \\
100 \% 30 \mathrm{~min}\end{array}$ & $0.0579 \mathrm{~min}^{-1}$ & $\begin{array}{c}\text { Co } 0.07 \mathrm{mg} \mathrm{L}^{-1} \\
\mathrm{pH}=6.0\end{array}$ \\
\hline
\end{tabular}


1. Della Longa, S.; Arcovito, A.; Girasole, M.; Hazemann, J.; Benfatto, M. Quantitative analysis of x-ray absorption near edge structure data by a full multiple scattering procedure: the $\mathrm{Fe}-\mathrm{CO}$ geometry in photolyzed carbonmonoxy-myoglobin single crystal. Phys. Rev. Lett. 2001, 87, 155501; DOI 10.1103/PhysRevLett.87.155501.

2. Cardelli, A.; Cibin, G.; Benfatto, M.; Della Longa, S.; Brigatti, M.; Marcelli, A. A crystal-chemical investigation of $\mathrm{Cr}$ substitution in muscovite by XANES spectroscopy. Phys. Chem. Miner. 2003, 30, 54-58; DOI 10.1007/s00269-002-0289-8.

3. Natoli, C.; Benfatto, M. A unifying scheme of interpretation of x-ray absorption-spectra based on the multiple-scattering theory. J. Phys. Colloq. 1986, 47, 11-23; DOI 10.1051/jphyscol:1986802.

4. Wu, Z.; Ouvard, G.; Lemaux, S.; Moreau, P.; Fressier, P.; Lemoigno, F.; Rouxel, J. Sulfur K-edge x-ray-absorption study of the charge transfer upon lithium intercalation into titanium disulfide. Phys. Rev. Lett. 1996, 77, 2101-2104; DOI 10.1103/PhyRevLett.77.2101.

5. Jawad, A.; Li, Y.; Lu, X.; Chen, Z.; Liu, W.; Yin, G.; Controlled leaching with prolonged activity for Co-LDH supported catalyst during treatment of organic dyes using bicarbonate activation of hydrogen peroxide. J. Hazard. Mater. 2015, 289, 165173; DOI 10.1016/j.jhazmat.2015.02.056. 
6. Luo, L.; Wang, Y.; Zhu, M.; Cheng, X.; Zhang, X.; Meng, X.; Huang, X.; Hao, $\mathrm{X}$. Co-Cu-Al layered double oxides as heterogeneous catalyst for enhanced degradation of organic pollutants in wastewater by activating peroxymonosulfate: Performance and synergistic effect. Ind. Eng. Chem. Res. 2019, 58 (20), 8699-8711; DOI 10.1021/acs.iecr.9b00167.

7. Hong, Y.; Peng, J.; Zhao, X.; Yan, Y.; Lai, B.; Yao, G. Efficient degradation of atrazine by $\mathrm{CoMgAl}$ layered double oxides catalyzed peroxymonosulfate: Optimization, degradation pathways and mechanism. Chem. Eng. J. 2019, 370, 354-363; DOI 10.1016/j.cej.2019.03.127.

8. Jawad, A.; Lang, J.; Liao, Z.; Khan, A.; Ifthikar, J.; Lv, Z.; Long, S.; Chen, Z.; Chen, Z. Activation of persulfate by CuOx@Co-LDH: A novel heterogeneous system for contaminant degradation with broad $\mathrm{pH}$ window and controlled leaching. Chem. Eng. J. 2018, 335, 548-559; DOI 10.1016/j.cej.2017.10.097.

9. Zhao, X.; Niu, G.; Zhang, L.; Guo, H.; Wen, X.; Liang, C.; Zeng, G. Co-Mn layered double hydroxide as an effective heterogeneous catalyst for degradation of organic dyes by activation of peroxymonosulfate. Chemosphere 2018, 204, 11-21; DOI 10.1016/j.chemosphere.2018.04.023.

10. Li, X.; Huang, X.; Xi, S.; Miao, S.; Ding, J.; Cai, W.; Liu, S.; Yang, X.; Yang, H.; Gao, J. Single cobalt atoms anchored on porous N-doped graphene with dual 
reaction sites for efficient Fenton-like catalysis. J. Am. Chem. Soc. 2018, 140 (39), 12469-12475; DOI 10.1021/jacs.8b05992.

11. Chu, C.; Yang, J.; Zhou, X.; Huang, D.; Qi, H.; Weon, S.; Li, J.; Elimelech, M.; Wang, A.; Kim, J. Cobalt single atoms on tetrapyridomacrocyclic support for efficient peroxymonosulfate activation. Environ. Sci. Technol. 2020, DOI 10.1021/acs.est.0c06086. 